\title{
An Inventory Model with Finite Replenishment Rate, Trade Credit Policy and Price-Discount Offer
}

\author{
Biswajit Sarkar, ${ }^{1}$ Shib Sankar Sana, ${ }^{2}$ and Kripasindhu Chaudhuri ${ }^{3}$ \\ ${ }^{1}$ Department of Applied Mathematics with Oceanology and Computer Programming, Vidyasagar University, Midnapore 721-102, India \\ ${ }^{2}$ Department of Mathematics, Bhangar Mahavidyalaya, University of Calcutta, Kolkata 743-502, India \\ ${ }^{3}$ Department of Mathematics, Jadavpur University, Kolkata 700-032, India
}

Correspondence should be addressed to Biswajit Sarkar; bsbiswajitsarkar@gmail.com

Received 25 December 2012; Revised 19 May 2013; Accepted 22 May 2013

Academic Editor: Paul C. Xirouchakis

Copyright (C) 2013 Biswajit Sarkar et al. This is an open access article distributed under the Creative Commons Attribution License, which permits unrestricted use, distribution, and reproduction in any medium, provided the original work is properly cited.

\begin{abstract}
When some suppliers offer trade credit periods and price discounts to retailers in order to increase the demand of their products, retailers have to face different types of discount offers and credits within which they have to take a decision which is the best offer for them to make more profit. The retailers try to buy perfect-quality items at a reasonable price, and also they try to invest returns obtained by selling those items in such a manner that their business is not hampered. In this point of view, we consider an economic order quantity (EOQ) model for various types of time-dependent demand when delay in payment and price discount are permitted by suppliers to retailers. The models of various demand patterns are discussed analytically. Some numerical examples and graphical representations are considered to illustrate the model.
\end{abstract}

\section{Introduction}

Many classical inventory models assume that demand is constant. In present marketing environment, few items follow constant demand. Many product's demands follow variable time-varying demand. The recent trend of the marketing system is to provide more buy opportunities to the retailer by the supplier by offering different discounts. To take the discount opportunity, retailers prefer to buy more beyond their capacity of buying. As a result, the supplier has the opportunity to sell more for better earning. This is the benefit of the supplier. The classical inventory model does not consider the delay time concept or variable demand. The proposed model considers time-varying demand and delay in payments along with finite replenishment rate.

The basic well-known square root formula for the EOQ of the item was formulated by Harris [1] based on constant demand. Donaldson [2] extended the constant demand to linear time-dependent demand model analytically with finite time horizon. Following Donaldson [2], significant contribution in this direction came out from researchers like Goyal [3], Goswami and Chaudhuri [4], Goyal et al. [5], and others.
Hariga and Benkherouf [6] discussed an optimal and heuristic replenishment model for deteriorating items with an exponentially time-varying demand. Wee [7] studied a deterministic lot size inventory model for deteriorating items with shortage and decline market. Khanra and Chaudhuri [8] extended an inventory model with quadratic increasing demand over a finite time horizon and shortages. Sana and Chaudhuri [9] studied an inventory model with linear trend demand incorporating shortages. Cárdenas-Barrón [10] discussed the derivation of inventory models by using analytic geometry and algebra. Sarkar et al. [11] explained an inventory model with quadratic time-varying demand by considering Euler-Lagrange method.

It is common to all that every customer prefers to buy more at reduced price. Some researchers like Abad [12], and Kim and Hwang [13] developed the traditional quantity discount model. In the traditional EOQ model, it was assumed that the retailer pays the purchasing cost when he received the items from a supplier. In trade-credit policy, the supplier allows a certain fixed period to pay the purchasing cost. This fixed period which is settled by the supplier is called the credit period to the retailer. During this credit period, 
TABLE 1: Comparisons of this model with previous works.

\begin{tabular}{|c|c|c|c|c|}
\hline Author/authors & Linear/quadratic demand & Exponential demand & Constant demand & Tread credit policy \\
\hline Harris [1] & & & $\checkmark$ & \\
\hline Donaldson [2] & $\checkmark$ & & & \\
\hline Goyal [14] & & & $\checkmark$ & $\checkmark$ \\
\hline Goyal [3] & $\checkmark$ & & & \\
\hline Goswami and Chaudhuri [4] & $\checkmark$ & & & \\
\hline Hariga and Benkherouf [6] & & $\checkmark$ & & $\checkmark$ \\
\hline Teng [18] & & & $\checkmark$ & $\checkmark$ \\
\hline Khanra and Chaudhuri [8] & $\checkmark$ & & & \\
\hline Arcelus et al. [19] & & & $\checkmark$ & $\checkmark$ \\
\hline Sana and Chaudhuri [9] & $\checkmark$ & & & \\
\hline Huang [20] & & & $\checkmark$ & $\checkmark$ \\
\hline Huang [21] & & & $\checkmark$ & $\checkmark$ \\
\hline Cárdenas-Barrón [10] & & & $\checkmark$ & $\checkmark$ \\
\hline Teng et al. [23] & & & $\checkmark$ & $\checkmark$ \\
\hline Sarkar [24] & $\checkmark$ & & & $\checkmark$ \\
\hline This paper & $\checkmark$ & $\checkmark$ & $\checkmark$ & $\checkmark$ \\
\hline
\end{tabular}

the supplier sells items to the retailer with different types of discounts to obtain more profit as early as possible during the credit period. Depending on this policy, Goyal [14] first developed an inventory model with permissible delay in payments. Aggarwal and Jaggi [15] developed an inventory model with an exponentially deteriorating rate by considering permissible delay in payments. Chu et al. [16] extended Goyal's [14] model by considering the case of deterioration. Jamal et al. [17] extended an inventory model with shortages.

Teng [18] developed an EOQ model for a retailer to order small lot size in order to take the benefit of permissible delay in payments. Arcelus et al. [19] developed an inventory model by considering the retailer's maximizing profit and inventory policies for vendor's trade promotion offer of price/credit on the purchase of perishable items. Huang [20] extended an inventory model of retailer's inventory system as a cost minimization model to determine the retailer's optimum inventory cycle time and optimal order quantity. Huang [21] developed an economic production quantity (EPQ) model of retailer's inventory system to investigate the optimal retailer's decisions under two levels of trade credit policy. CárdenasBarrón [22] extended optimal ordering policies in response to a discount offer. Teng et al. [23] explained optimal ordering decisions with returns and excess inventory. Sarkar [24] discussed an inventory model with delay in payments in the presence of imperfect production. Sarkar [25] developed an inventory model with delay in payments and time-varying deterioration rate. Forghani et al. [26] explained an inventory model in the single period inventory system with price adjustment.

This paper considers an inventory model for credit periods and price-discount offers with different types of time varying demand and constant supply rate $K$ up to time $t=t_{1}$. During $\left[0, t_{1}\right]$, inventory piles up by adjusting the demand. The accumulated inventory level at time $t_{1}$ depletes gradually to meet the demand, and the level reaches zero level at time
$T\left(t_{1} \leq T\right)$. The agreement between the supplier and the retailer is such that total purchasing cost of whole amount $\left(K t_{1}\right)$ would be paid within the time $R\left(R>t_{1}\right)$ with purchasing cost at discount rate $\rho$. The different delay periods with different discount rates on the purchasing cost are permitted by the supplier to the retailer. During the credit period, the retailer can earn interest by selling items whereas interest of purchasing cost is charged against the delay of excess time of credit of payment period by the retailer to the supplier.

The rest of the paper is designed as follows. The mathematical model is presented in Section 2. In Section 3, numerical examples are given. Finally, concluding remarks are explained in Section 4. See Table 1 for the comparison of this model with previous works.

\section{Mathematical Model}

We consider the following notation to develop the model.

$T^{*}$ : the optimal length of inventory cycle (decision variable)

$t_{1}^{*}$ : the optimal duration of replenishment (decision variable)

$Q_{1}(t)$ : on-hand inventory at time $t\left(0 \leq t \leq t_{1}\right)$

$Q_{2}(t)$ : on-hand inventory at time $t\left(t_{1} \leq t \leq T\right)$

$D(t)$ : time-varying demand rate

$K$ : constant replenishment/supply rate

$R$ : variable delay period

$R_{i}: i$ th permissible delay period

$\rho_{i}$ : discount rate on purchasing cost at $i$ th permissible delay period

$C_{1}$ : ordering cost per order 
$\mathrm{C}_{2}$ : unit holding cost per unit time, excluding interest charge

$\mathrm{C}_{3}$ : purchasing cost per unit

$\mathrm{C}_{4}$ : maximum retail price per unit

$P$ : selling price per unit

$I_{c}$ : rate of interest gaining due to the credit balance

$I_{f}$ : rate of interest due to financing inventory

$T$ : length of the inventory cycle

$t_{1}$ : duration of the replenishment

$Z_{1 i}$ : average profit of the system when $T \geq R_{i}$

$Z_{2 i}$ : average profit of the system when $T \leq R_{i}$.

The following assumptions are considered to develop this model.

(1) The inventory system involves only single type of product.

(2) The demand rate is constant or time dependent (quadratic, linear, and exponential).

(3) Different discount rates on the purchasing cost for different delay periods are considered.

(4) Replenishment rate is instantaneously infinite, but its size is finite.

(5) Time horizon is infinite.

(6) Lead time is negligible.

(7) Neither shortage nor backlogging is considered.

The cycle starts with zero inventory at supply rate $K$. The replenishment or supply continues up to time $t_{1}$. During the time span $\left[0, t_{1}\right]$, inventory piles up by adjusting the demand in the market. This accumulated inventory level at time $t_{1}$ depletes gradually to meet the demand and it reaches zero level at time $t=T\left(T>t_{1}\right)$. Generally, the supplier offers delay period $R\left(R>t_{1}\right)$ to the retailer to pay the total purchasing cost $\left(C_{3} K t_{1}\right)$ of items. For different delay periods $R_{i}(i=1,2,3)$, different discounts $\rho_{i}(i=1,2,3)$ of purchasing cost are offered to the retailer by the supplier. In this direction, we consider the purchasing cost of different delay periods as follows:

$$
C_{3}= \begin{cases}C_{4}\left(1-\rho_{1}\right) & \text { when } R=R_{1}, \\ C_{4}\left(1-\rho_{2}\right) & \text { when } R=R_{2}, \\ C_{4}\left(1-\rho_{3}\right) & \text { when } R=R_{3}, \\ \infty & \text { when } R>R_{3}\end{cases}
$$

where $R_{i}^{\prime}$ s are the $i$ th permissible delay to settle the purchasing cost at which the discount rate to the retailer is $\rho_{i}$. Also $C_{3}$ tends to be $\infty$ at $R>R_{3}$. That is, at infinite purchasing cost, the retailer never purchases any item from the supplier. Indirectly, the supplier would not supply the product to the retailer while delay period $R$ exceeds $R_{3}$. In our model two cases may arise.

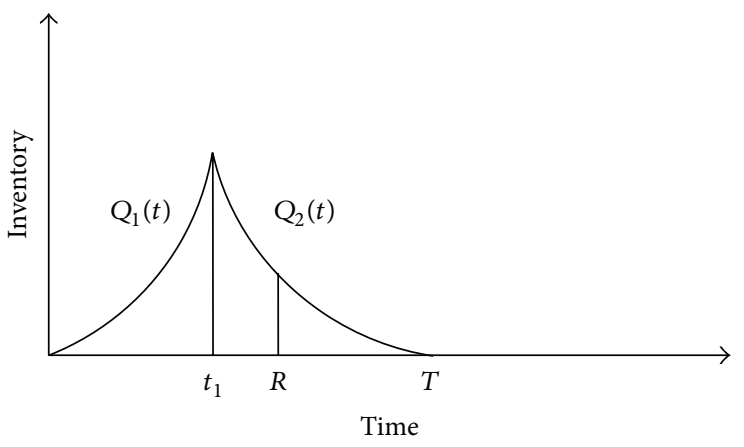

FIgURE 1: Inventory versus time (Case $1 T \geq R$ ).

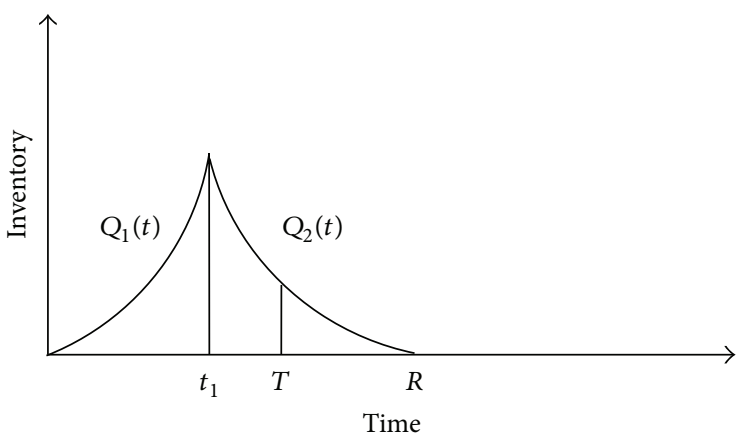

Figure 2: Inventory versus time (Case $2 T \geq R$ ).

Case $1(T \geq R)$. That is, inventory cycle length $T$ is larger or equal to the credit period $R$ (see Figure 1 ). When $T \geq R$, there are some profits based on credit balance during the delay period and there is some interest charged due to financing inventory during $[R, T]$.

Case $2(T \leq R)$. That is, inventory cycle length $T$ is smaller or equal to the credit period $R$ (see Figure 2). When $T \leq R$, there are some profits based on credit balance during the delay period and there is no interest charged due to financing inventory.

The governing differential equations of this model are

$$
\begin{gathered}
\frac{d Q_{1}}{d t}=K-D(t) \quad \text { with } Q_{1}(0)=0,0 \leq t \leq t_{1}, \\
\frac{d Q_{2}}{d t}=-D(t) \quad \text { with } Q_{2}(T)=0, t_{1} \leq t \leq T .
\end{gathered}
$$

From (2), we obtain

$$
\begin{gathered}
Q_{1}(t)=K t-\int_{0}^{t} D(t) d t, \quad 0 \leq t \leq t_{1}, \\
Q_{2}(t)=\int_{t}^{T} D(t) d t, \quad t_{1} \leq t \leq T .
\end{gathered}
$$

By utilizing the continuity at $t_{1}, Q_{1}\left(t_{1}\right)=Q_{2}\left(t_{1}\right)$, we have

$$
K t_{1}-\int_{0}^{t_{1}} D(t) d t=\int_{t_{1}}^{T} D(t) d t,
$$


that is,

$$
t_{1}=\frac{1}{K} \int_{0}^{T} D(t) d t
$$

Now we formulate Cases 1 and 2 using (3) to (6).

Case $1(T \geq R)$. When the inventory cycle $(T)$ is larger or equal to the credit period $R$, the holding cost excluding the interest charges is $C_{2}\left\{\int_{0}^{t_{1}} Q_{1}(t) d t+\int_{t_{1}}^{T} Q_{2}(t) d t\right\}$.

The profit gains due to credit balance during the delay period $[0, R]$ are $I_{c} P \int_{0}^{R}(R-t) D(t) d t$.

The interest charged for financing inventory during $[R, T]$ is $I_{f} C_{3} \int_{R}^{T} Q_{2}(t) d t$.

Therefore, the total profit is (see Figure 1)

$$
\begin{aligned}
P_{1 i}=[( & \left.P-C_{3}\right) K t_{1} \\
& +I_{c} P\left\{\int_{0}^{t_{1}}\left(R_{i}-t\right) D(t) d t+\int_{t_{1}}^{R_{i}}\left(R_{i}-t\right) D(t) d t\right\} \\
& -C_{2}\left\{\int_{0}^{t_{1}} Q_{1}(t) d t+\int_{t_{1}}^{T} Q_{2}(t) d t\right\} \\
& \left.-I_{f} C_{3} \int_{R_{i}}^{T} Q_{2}(t) d t-C_{1}\right] \text { for } i \in\{1,2,3\} .
\end{aligned}
$$

Hence, the average profit is

$$
\begin{aligned}
Z_{1 i}=\frac{P_{1 i}}{T} & \\
=\frac{1}{T}[ & \left(P-C_{3}\right) K t_{1} \\
& +I_{c} P\left\{\int_{0}^{t_{1}}\left(R_{i}-t\right) D(t) d t+\int_{t_{1}}^{R_{i}}\left(R_{i}-t\right) D(t) d t\right\} \\
& -C_{2}\left\{\int_{0}^{t_{1}} Q_{1}(t) d t+\int_{t_{1}}^{T} Q_{2}(t) d t\right\} \\
& \left.\quad-I_{f} C_{3} \int_{R_{i}}^{T} Q_{2}(t) d t-C_{1}\right] \text { for } i \in\{1,2,3\} .
\end{aligned}
$$

Case $2(T \leq R)$. When the inventory cycle $(T)$ is smaller or equal to the credit period $R$, the holding cost excluding the interest charges is $C_{2}\left\{\int_{0}^{t_{1}} Q_{1}(t) d t+\int_{t_{1}}^{T} Q_{2}(t) d t\right\}$.

The profit gains due to credit balance during the time $[0, R]$ are $I_{c} P\left[\int_{0}^{T}(T-t) D(t) d t+K t_{1}(R-T)\right]$.
Therefore, the total profit is (see Figure 2)

$$
\begin{aligned}
& P_{2 i}=[\left(P-C_{3}\right) K t_{1} \\
&+I_{c} P\left\{\int_{0}^{t_{1}}(T-t) D(t) d t\right. \\
&\left.+\int_{t_{1}}^{T}(T-t) D(t) d t+K t_{1}\left(R_{i}-T\right)\right\} \\
&\left.-C_{2}\left\{\int_{0}^{t_{1}} Q_{1}(t) d t+\int_{t_{1}}^{T} Q_{2}(t) d t\right\}-C_{1}\right] \\
& \text { for } i \in\{1,2,3\} .
\end{aligned}
$$

Hence, the average profit is

$$
\begin{aligned}
Z_{2 i}= & \frac{P_{2 i}}{T} \\
=\frac{1}{T}\left[\left(P-C_{3}\right) K t_{1}\right. & \\
& +I_{c} P\left\{\int_{0}^{t_{1}}(T-t) D(t) d t\right. \\
& \left.+\int_{t_{1}}^{T}(T-t) D(t) d t+K t_{1}\left(R_{i}-T\right)\right\}
\end{aligned}
$$$$
\left.-C_{2}\left\{\int_{0}^{t_{1}} Q_{1}(t) d t+\int_{t_{1}}^{T} Q_{2}(t) d t\right\}-C_{1}\right]
$$$$
\text { for } i \in\{1,2,3\} \text {. }
$$

Now our objective is to maximize $Z=\operatorname{Sup}\left\{Z_{1 i}, Z_{2 i}\right\}$ for $i \in\{1,2,3\}$ and obtain the optimal replenishment period $t_{1}^{*}$ and inventory cycle length $T^{*}$. We discuss various timedependent or constant demands by utilizing this general formula.

2.1. Quadratic Demand Pattern. The quadratic time-dependent demand is of the form $D(t)=a+b t+c t^{2}$ where $a>0$, $b>0$, and $c>0$. This trend of demand is applied to the products like essential commodities and seasonal goods.

From (2), we have

$$
\begin{gathered}
Q_{1}(t)=K t-a t-\frac{b t^{2}}{2}-\frac{c t^{3}}{3}, \\
Q_{2}(t)=a(T-t)+\frac{b}{2}\left(T^{2}-t^{2}\right)+\frac{c}{3}\left(T^{3}-t^{3}\right) .
\end{gathered}
$$


Using these $Q_{1}(t)$ and $Q_{2}(t)$ in (8) and (10), we obtain

$$
\begin{aligned}
& Z_{1 i}=\frac{1}{T}\left[\left(P-C_{3}\right) K t_{1}\right. \\
& +I_{c} P\left\{\frac{a R_{i}^{2}}{2}+\frac{b R_{i}^{3}}{6}+\frac{c R_{i}^{4}}{12}\right\} \\
& -C_{2}\left\{\frac{K t_{1}^{2}}{2}+\frac{a T^{2}}{2}+\frac{b T^{3}}{3}+\frac{c T^{4}}{4}\right. \\
& \left.-a T t_{1}-\frac{b T^{2} t_{1}}{2}-\frac{c T^{3} t_{1}}{3}\right\} \\
& -I_{f} C_{3}\left\{\frac{a T^{2}}{2}+\frac{b T^{3}}{3}+\frac{c T^{4}}{4}\right. \\
& -a\left(T R_{i}-\frac{R_{i}^{2}}{2}\right)-\frac{b}{2}\left(T^{2} R_{i}-\frac{R_{i}^{3}}{3}\right) \\
& \left.\left.-\frac{c}{3}\left(T^{3} R_{i}-\frac{R_{i}^{4}}{4}\right)\right\}-C_{1}\right] \text {, } \\
& Z_{2 i}=\frac{1}{T}\left[\left(P-C_{3}\right) K t_{1}\right. \\
& +I_{c} P\left\{\frac{a T^{2}}{2}+\frac{b T^{3}}{6}+\frac{c T^{4}}{12}+K t_{1}\left(R_{i}-T\right)\right\} \\
& -C_{2}\left\{\frac{K t_{1}^{2}}{2}+\frac{a T^{2}}{2}+\frac{b T^{3}}{3}\right. \\
& \left.\left.+\frac{c T^{4}}{4}-a T t_{1}-\frac{b T^{2} t_{1}}{2}-\frac{c T^{3} t_{1}}{3}\right\}-C_{1}\right] .
\end{aligned}
$$

Using (6) in the above objective functions, we obtain

$$
\begin{aligned}
& Z_{1 i}=\left[\left(P-C_{3}\right)\left(a+\frac{b T}{2}+\frac{c T^{2}}{3}\right)\right. \\
&+ \frac{I_{c} P}{T}\left\{\frac{a R_{i}^{2}}{2}+\frac{b R_{i}^{3}}{6}+\frac{c R_{i}^{4}}{12}\right\} \\
&-C_{2}\left\{\frac { 1 } { 2 K } \left(a^{2} T+\frac{b^{2} T^{3}}{4}+\frac{c^{2} T^{5}}{9}+a b T^{2}\right.\right. \\
&\left.+\frac{b c T^{4}}{3}+\frac{2 a c T^{3}}{3}\right)+\frac{a T}{2}+\frac{b T^{2}}{3} \\
&+\frac{c T^{3}}{4}-\frac{a^{2} T}{K}-\frac{a b T^{2}}{K} \\
&\left.-\frac{2 a c T^{3}}{3 K}-\frac{b^{2} T^{3}}{4 K}-\frac{b c T^{4}}{3 K}-\frac{c^{2} T^{5}}{9 K}\right\}
\end{aligned}
$$

$$
\begin{aligned}
& -I_{f} C_{3}\left\{\frac{a T}{2}+\frac{b T^{2}}{3}+\frac{c T^{3}}{4}-a R_{i}\right. \\
& +\frac{a R_{i}^{2}}{2 T}-\frac{b T R_{i}}{2}+\frac{b R_{i}^{3}}{6 T} \\
& \left.\left.-\frac{c T^{2} R_{i}}{3}+\frac{c R_{i}^{4}}{12 T}\right\}-\frac{C_{1}}{T}\right] \\
& Z_{2 i}=\left[\left(P-C_{3}\right)\left(a+\frac{b T}{2}+\frac{c T^{2}}{3}\right)\right. \\
& +I_{c} P\left\{a R_{i}+\frac{b T R_{i}}{2}+\frac{c T^{2} R_{i}}{3}-\frac{a T}{2}-\frac{b T^{2}}{3}-\frac{c T^{3}}{4}\right\} \\
& -C_{2}\left\{\frac { 1 } { 2 K } \left(a^{2} T+\frac{b^{2} T^{3}}{4}+\frac{c^{2} T^{5}}{9}+a b T^{2}\right.\right. \\
& \left.+\frac{b c T^{4}}{3}+\frac{2 a c T^{3}}{3}\right)+\frac{a T}{2}+\frac{b T^{2}}{3} \\
& +\frac{c T^{3}}{4}-\frac{a^{2} T}{K}-\frac{a b T^{2}}{K}-\frac{2 a c T^{3}}{3 K} \\
& \left.\left.-\frac{b^{2} T^{3}}{4 K}-\frac{b c T^{4}}{3 K}-\frac{c^{2} T^{5}}{9 K}\right\}-\frac{C_{1}}{T}\right] \text {. }
\end{aligned}
$$

To obtain the optimal cycle length, we construct two lemmas as follows.

Lemma 1. When $\left.\left(d Z_{1 i} / d T\right)\right|_{T=T^{*}}=0$ exists for $T^{*} \in\left[R_{i}, \infty\right)$, then $Z_{1 i}$ has a maximum value at $T=T^{*}$ if $\left[4 \xi_{1}(c)\left(T^{*}\right)^{6}+\right.$ $\left.\xi_{2}(b, c)\left(T^{*}\right)^{5}+\xi_{3}(a, b, c)\left(T^{*}\right)^{4}+\xi_{4}(a, b, c)\left(T^{*}\right)^{3}-2 \xi_{6}(a, b, c)\right]<$ 0 . Otherwise, $Z_{1 i}$ has a maximum value at unique $T^{*}=\{T \mid$ $\left.(c / 3) T^{3}+(b / 2) T^{2}+a T-K R_{i}=0, T>0\right\}$ if $\left[\xi_{1}(c) R_{i}^{6}+\right.$ $\xi_{2}(b, c) R_{i}^{5}+\xi_{3}(a, b, c) R_{i}^{4}+\xi_{4}(a, b, c) R_{i}^{3}+\xi_{5}(a, b) R_{i}^{2}+$ $\left.\xi_{6}(a, b, c)\right]>0$ holds.

\section{Proof. See Appendix A.}

Lemma 2. When $\left.\left(d Z_{2 i} / d T\right)\right|_{T=T^{*}}=0$ exists for $T^{*} \in\left[0, R_{i}\right]$, then $Z_{2 i}$ has a maximum value at $T=T^{*}$ if $\left[5\left(T^{*}\right)^{6} \xi_{1}(c)+\right.$ $\left.\left(T^{*}\right)^{5} \xi_{2}(b, c)+2\left(T^{*}\right)^{4} \xi_{7}(a, b, c)+\left(T^{*}\right)^{3} \xi_{8}(a, b, c)-2 C_{1}\right]<0$. Otherwise, $Z_{2 i}$ has a maximum value at $T=R_{i}$ if $\left[\left(R_{i}\right)^{6} \xi_{1}(c)+\right.$ $\left(R_{i}\right)^{5} \xi_{2}(b, c)+\left(R_{i}\right)^{4} \xi_{7}(a, b, c)+\left(R_{i}\right)^{3} \xi_{8}(a, b, c)+\left(R_{i}\right)^{2} \xi_{9}(a, b, c)+$ $\left.C_{1}\right]>0$.

Proof. See Appendix A.

2.2. Linear Time-Dependent Demand Pattern. We consider linear time-dependent demand which can be obtained if we substitute $c=0$ in the form of quadratic demand. We get $D(t)=a+b t, a>0, b>0$ (see for instances Donaldson [2], Goyal [3], and Goswami and Chaudhuri [4]). Generally, in some computer games, computer android applications, we obtain this type of linear time-dependent demand. 
Now from (2), we have

$$
Q_{1}(t)=K t-a t-\frac{b t^{2}}{2}
$$

and $Q_{2}(t)=a(T-t)+(b / 2)\left(T^{2}-t^{2}\right)$, respectively.

Using $Q_{1}(t)$ and $Q_{2}(t)$ in $(8)$ and $(10)$, we obtain

$$
\begin{aligned}
Z_{1 i}=\frac{1}{T}[ & \left(P-C_{3}\right) K t_{1} \\
& +I_{c} P\left\{\frac{a R_{i}^{2}}{2}+\frac{b R_{i}^{3}}{6}\right\} \\
& -C_{2}\left\{\frac{K t_{1}^{2}}{2}+\frac{a T^{2}}{2}+\frac{b T^{3}}{3}-a T t_{1}-\frac{b T^{2} t_{1}}{2}\right\} \\
& -I_{f} C_{3}\left\{\frac{a T^{2}}{2}+\frac{b T^{3}}{3}-a\left(T R_{i}-\frac{R_{i}^{2}}{2}\right)\right. \\
Z_{2 i}=\frac{1}{T}[ & \left.\left.\left(P-C_{3}\right) K t_{1}\left(T^{2} R_{i}-\frac{R_{i}^{3}}{3}\right)\right\}-C_{1}\right], \\
& +I_{c} P\left\{\frac{a T^{2}}{2}+\frac{b T^{3}}{6}+K t_{1}\left(R_{i}-T\right)\right\} \\
& \left.-C_{2}\left\{\frac{K t_{1}^{2}}{2}+\frac{a T^{2}}{2}+\frac{b T^{3}}{3}-a T t_{1}-\frac{b T^{2} t_{1}}{2}\right\}-C_{1}\right] .
\end{aligned}
$$

Using (6) in the above objective functions, we have

$$
\begin{aligned}
Z_{1 i}= & {\left[\left(P-C_{3}\right)\left(a+\frac{b T}{2}\right)+\frac{I_{c} P}{T}\left\{\frac{a R_{i}^{2}}{2}+\frac{b R_{i}^{3}}{6}\right\}\right.} \\
& -C_{2}\left\{\frac{1}{2 K}\left(a^{2} T+\frac{b^{2} T^{3}}{4}+a b T^{2}\right)\right. \\
& \left.+\frac{a T}{2}+\frac{b T^{2}}{3}-\frac{a^{2} T}{K}-\frac{a b T^{2}}{K}-\frac{b^{2} T^{3}}{4 K}\right\} \\
& -I_{f} C_{3}\left\{\frac{a T}{2}+\frac{b T^{2}}{3}-a R_{i}+\frac{a R_{i}^{2}}{2 T}\right. \\
Z_{2 i}= & \left.\left(P-\frac{b T R_{i}}{2}+\frac{b R_{i}^{3}}{6 T}\right\}-\frac{C_{1}}{T}\right], \\
& +C_{c} P\left\{a+\frac{b T}{2}\right)\left(a R_{i}+\frac{b T R_{i}}{2}-\frac{a T}{2}-\frac{b T^{2}}{3}\right\}
\end{aligned}
$$

$$
\begin{aligned}
-C_{2}\{ & \frac{1}{2 K}\left(a^{2} T+\frac{b^{2} T^{3}}{4}+a b T^{2}\right) \\
& +\frac{a T}{2}+\frac{b T^{2}}{3}-\frac{a^{2} T}{K} \\
& \left.\left.-\frac{a b T^{2}}{K}-\frac{b^{2} T^{3}}{4 K}\right\}-\frac{C_{1}}{T}\right] .
\end{aligned}
$$

To obtain the optimal cycle length, we construct the following lemmas.

Lemma 3. When $\left.\left(d Z_{1 i} / d T\right)\right|_{T=T^{*}}=0$ exists for $T^{*} \in\left[R_{i}, \infty\right)$, then $Z_{1 i}$ has a maximum value at $T=T^{*}$ if $\left[2\left(T^{*}\right)^{4} \xi_{3}(a, b, 0)+\right.$ $\left.\left(T^{*}\right)^{3} \xi_{4}(a, b, 0)-2 \xi_{6}(a, b, 0)\right]<0$. Otherwise $Z_{1 i}$ has a global maximum value at $T=\left((-a)+\left(\sqrt{a^{2}+2 K b R_{i}}\right)\right) / b$ if $R_{i} \leq$ $2(K-a) / b$ holds.

Proof. See Appendix B.

Lemma 4. When $\left.\left(d Z_{2 i} / d T\right)\right|_{T=T^{*}}=0$ exists for $T^{*} \in\left[0, R_{i}\right]$, then $Z_{2 i}$ has a maximum value at $T=T^{*}$ if $\left[2\left(T^{*}\right)^{4} \xi_{3}(a, b, 0)+\right.$ $\left.\left(T^{*}\right)^{3} \xi_{8}(a, b, 0)-2 C_{1}\right]<0$. Otherwise $Z_{2 i}$ has a maximum value at $T=R_{i}$ if $\left[\left(R_{i}\right)^{4} \xi_{3}(a, b, 0)+\left(R_{i}\right)^{3} \xi_{8}(a, b, 0)+\right.$ $\left.\left(R_{i}\right)^{2} \xi_{5}(a, b)+C_{1}\right]>0$.

\section{Proof. See Appendix B.}

2.3. Constant Demand Pattern. We consider the demand as constant which can be found by substituting $b=0$ in the linear demand. We obtain $D=a, a>0$. See for instances Harris [1] and Cárdenas-Barrón [10, 22]. This type of demand is usually found in product's life cycle.

Now from (2), we have $Q_{1}(t)=(K-a) t$ and $Q_{2}(t)=$ $a(T-t)$, respectively.

Using $Q_{1}(t)$ and $Q_{2}(t)$ in (8) and (10), we get

$$
\begin{aligned}
& Z_{1 i}=\frac{1}{T}[\left(P-C_{3}\right) K t_{1}+I_{c} P\left(\frac{a R_{i}^{2}}{2}\right) \\
&-C_{2}\left\{\frac{K t_{1}^{2}}{2}+\frac{a T^{2}}{2}-a T t_{1}\right\} \\
&\left.-I_{f} C_{3}\left\{\frac{a T^{2}}{2}-a\left(T R_{i}-\frac{R_{i}^{2}}{2}\right)\right\}-C_{1}\right], \\
& Z_{2 i}=\frac{1}{T}\left[\left(P-C_{3}\right) K t_{1}+I_{c} P\left\{\frac{a T^{2}}{2}+K t_{1}\left(R_{i}-T\right)\right\}\right. \\
&\left.\quad-C_{2}\left\{\frac{K t_{1}^{2}}{2}+\frac{a T^{2}}{2}-a T t_{1}\right\}-C_{1}\right],
\end{aligned}
$$

respectively. Using (6) in the above functions, we obtain

$$
\begin{aligned}
Z_{1 i}= & {\left[\left(P-C_{3}\right) a+\frac{I_{c} P a R_{i}^{2}}{2 T}-C_{2}\left\{\frac{a T}{2}-\frac{a^{2} T}{2 K}\right\}\right.} \\
& \left.-I_{f} C_{3}\left\{\frac{a T}{2}-a R_{i}+\frac{a R_{i}^{2}}{2 T}\right\}-\frac{C_{1}}{T}\right],
\end{aligned}
$$




$$
\begin{array}{r}
Z_{2 i}=\left[\left(P-C_{3}\right)(a)+I_{c} P\left\{a R_{i}-\frac{a T}{2}\right\}\right. \\
\left.-C_{2}\left\{\frac{a T}{2}-\frac{a^{2} T}{2 K}\right\}-\frac{C_{1}}{T}\right] .
\end{array}
$$

To obtain optimal cycle length, we formulate the following lemmas.

Lemma 5. If the conditions $R_{i}^{2}<K\left\{2 C_{1}+a R_{i}^{2}\left(I_{f} C_{3}-I_{c} P\right)\right\} /$ $\left(C_{2} a(K-a)+I_{f} C_{3} a K\right)<K^{2} R_{i}^{2} / a^{2}$ and $K\left\{2 C_{1}+a R_{i}^{2}\left(I_{f} C_{3}-\right.\right.$ $\left.\left.I_{c} P\right)\right\}>0$ hold, then $Z_{1 i}$ has a global maximum at $T=T^{*}=$ $\left[K\left\{2 C_{1}+a R_{i}^{2}\left(I_{f} C_{3}-I_{c} P\right)\right\} /\left(C_{2} a(K-a)+I_{f} C_{3} a K\right)\right]^{1 / 2}$.

Proof. See Appendix C.

Lemma 6. If $0<2 C_{1} K /\left(C_{2} a(K-a)-I_{c} P a K\right)<R_{i}^{2}$ hold, then $Z_{2 i}$ has a global maximum at $T=T^{*}=\left[2 C_{1} K /\left(C_{2} a(K-a)-\right.\right.$ $\left.\left.I_{c} \mathrm{PaK}\right)\right]^{1 / 2}$.

Proof. See Appendix C.

2.4. Exponential Demand Pattern. We consider another important type of demand as exponential type, that is, $D(t)$ varies exponentially with time $t$. In this case, the demand rate increases very fast as in seasonal goods, new computer parts like RAM and data storage device. For this type of demand rate, we consider $D(t)=a \exp (b t)$ where $a>0, b>0$.

From (2), we have

$$
\begin{aligned}
Q_{1}(t) & =K t+\frac{a}{b}\{1-\exp (b t)\}, \\
Q_{2}(t) & =\frac{a}{b}\{\exp (b T)-\exp (b t)\} .
\end{aligned}
$$

Using these $Q_{1}(t)$ and $Q_{2}(t)$ in (8) and (10), we obtain

$$
\begin{aligned}
Z_{1 i}=\frac{1}{T}[ & \left(P-C_{3}\right) K t_{1}-I_{c} P\left\{\frac{a R_{i}}{b}+\frac{a}{b^{2}}\left(1-\exp \left(b R_{i}\right)\right)\right\} \\
& -C_{2}\left\{\frac{K t_{1}^{2}}{2}+\frac{a}{b}\left(t_{1}+\frac{1}{b}+T \exp (b T)\right.\right. \\
\left.\left.-\frac{\exp (b T)}{b}-t_{1} \exp (b T)\right)\right\} & -I_{f} C_{3} \frac{a}{b}\left\{\left(T-R_{i}\right) \exp (b T)\right. \\
\left.\left.-\frac{\exp (b T)-\exp \left(b R_{i}\right)}{b}\right\}-C_{1}\right] & \\
Z_{2 i}=\frac{1}{T}[( & \left.P-C_{3}\right) K t_{1} \\
& -I_{c} P\left\{\frac{a T}{b}+\frac{a}{b^{2}}(1-\exp (b T))-K t_{1}\left(R_{i}-T\right)\right\}
\end{aligned}
$$

$$
\begin{gathered}
-C_{2}\left\{\frac{K t_{1}^{2}}{2}+\frac{a}{b}\left(t_{1}+\frac{1}{b}+T \exp (b T)-\frac{\exp (b T)}{b}\right.\right. \\
\left.\left.\left.-t_{1} \exp (b T)\right)\right\}-C_{1}\right] .
\end{gathered}
$$

From (6), we have

$$
t_{1}=\frac{1}{K} \int_{0}^{T} a \exp (b t) d t=\frac{a}{K b}[\exp (b T)-1] .
$$

Substituting this in $Z_{1 i}$ and $Z_{2 i}$, we have

$$
\begin{aligned}
& Z_{1 i}=\frac{a}{b T}[\left(P-C_{3}\right)(\exp (b T)-1) \\
&-I_{c} P\left\{R_{i}+\frac{1}{b}\left(1-\exp \left(b R_{i}\right)\right)\right\} \\
&- C_{2}\left\{\frac{1}{b}+T \exp (b T)-\frac{\exp (b T)}{b}+\frac{a \exp (b T)}{K b}\right. \\
&\left.-\frac{a \exp (2 b T)}{2 K b}-\frac{a}{2 K b}\right\} \\
&- I_{f} C_{3}\left\{\left(T-R_{i}\right) \exp (b T)\right. \\
&\left.\left.-\frac{\exp (b T)-\exp \left(b R_{i}\right)}{b}\right\}-\frac{b C_{1}}{a}\right], \\
& Z_{2 i}=\frac{a}{b T}[(\left.P-C_{3}\right)(\exp (b T)-1) \\
&- I_{c} P\left\{\frac{1-\exp (b T)}{b}+R_{i}+\left(T-R_{i}\right) \exp (b T)\right\} \\
&- C_{2}\left\{\frac{1}{b}+T \exp (b T)-\frac{\exp (b T)}{b}+\frac{a \exp (b T)}{K b}\right. \\
&\left.\left.\left.-\frac{a \exp (2 b T)}{2 K b}-\frac{a}{2 K b}\right)\right\}-\frac{b C_{1}}{a}\right] .
\end{aligned}
$$

Lemma 7. When $\left.\left(d Z_{1 i} / d T\right)\right|_{T=T^{*}}=0$ exists for $T^{*} \epsilon$ $\left[R_{i}, \infty\right)$, then $Z_{1 i}$ has a maximum value at $T=T^{*}$ if $\left(d^{2} Z_{1 i} /\right.$ $\left.d T^{2}\right)\left.\right|_{T=T^{*}}<0$. Otherwise, $Z_{1 i}$ has a unique maximum value $T=T^{*}=(1 / b)\left[\ln \left(1+K b R_{i} / a\right)\right]$, if $\left.\left(d Z_{1 i} / d T\right)\right|_{T=R_{i}}>0$.

Proof. See Appendix D.

Lemma 8. When $\left.\left(d Z_{2 i} / d T\right)\right|_{T=T^{*}}=0$ exists for $T^{*} \in$ $\left[0, R_{i}\right]$, then $Z_{2 i}$ has a maximum value at $T=T^{*}$ if $\left(d^{2} Z_{2 i} /\right.$ $\left.d T^{2}\right)\left.\right|_{T=T^{*}}<0$. Otherwise, $Z_{2 i}$ has a maximum value $T=R_{i}$, if $\left.\left(d Z_{2 i} / d T\right)\right|_{T=R_{i}}>0$

Proof. See Appendix D.

\section{Numerical Examples}

Example A. We consider the following parametric values in appropriate units: $C_{1}=\$ 250$ per order, $C_{2}=\$ 20 /$ unit $/$ year, 


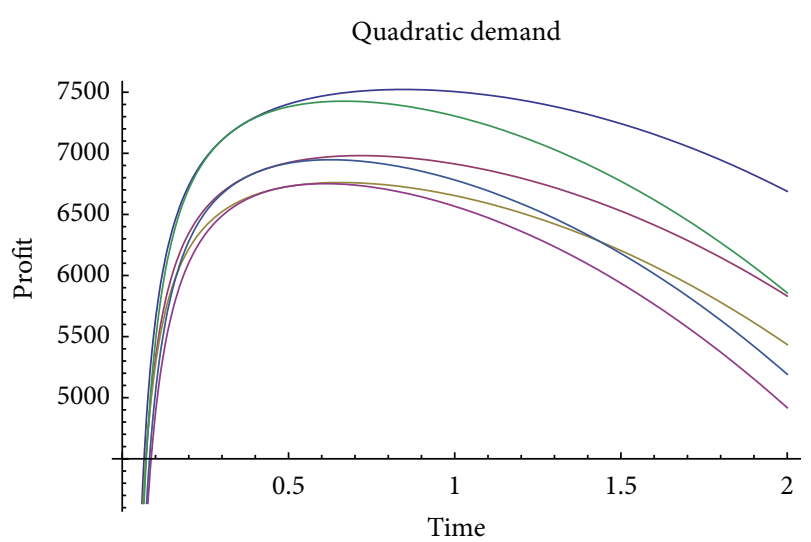

FIGURE 3: The figures of the average profit functions are in descending order as $Z_{11}, Z_{21}, Z_{12}, Z_{22}, Z_{13}$, and $Z_{23}$.

$R_{1}=120 / 365$ year, $R_{2}=150 / 365$ year, $R_{3}=180 / 365$ year, $\rho_{1}=10 \%, \rho_{2}=5 \%, \rho_{3}=2 \%, a=100$ units, $b=50$ units, $c=$ 10 units, $D(t)=a+b t+c t^{2}, C_{4}=\$ 120 /$ units, $P=\$ 180 /$ units, $I_{f}=0.16 / \$ /$ year, $I_{c}=0.13 / \$ /$ year, and $K=500$ units.

Then the optimal solutions are $\left\{Z_{11}=\$ 7522.33, T^{*}=\right.$ 0.85 year, $t_{1}^{*}=0.21$ year $\},\left\{Z_{12}=\$ 6980.84, T^{*}=0.72\right.$ year, $t_{1}^{*}=0.17$ year $\},\left\{Z_{13}=\$ 6760.68, T^{*}=0.66\right.$ year, $t_{1}^{*}=$ 0.15 year $\},\left\{Z_{21}=\$ 7174.54, T^{*}=0.33\right.$ year, $t_{1}^{*}=0.07$ year $\}$, $\left\{Z_{22}=\$ 6852.25, T^{*}=0.41\right.$ year, $t_{1}^{*}=0.91$ year $\},\left\{Z_{23}=\right.$ $\$ 6725.72, T^{*}=0.49$ year, $t_{1}^{*}=0.11$ year $\}$.

Among the above optimal solutions, the optimal solution is $\left\{Z_{11}=\$ 7522.33, T^{*}=0.85\right.$ year, $t_{1}^{*}=0.21$ year $\}$ global maximum. The average profit function is highly nonlinear. Figure 3 shows the concavity of the functions and comparisons of the cost functions.

Example B. We consider the following parametric values in appropriate units: $C_{1}=\$ 250$ per order, $C_{2}=\$ 20 /$ unit $/$ year, $R_{1}=120 / 365$ year, $R_{2}=150 / 365$ year, $R_{3}=180 / 365$ year, $\rho_{1}=10 \%, \rho_{2}=5 \%, \rho_{3}=2 \%, a=100$ units, $b=50$ units, $D(t)=a+b t, C_{4}=\$ 120 /$ units, $P=\$ 180 /$ units, $I_{f}=0.16 / \$ /$ year, $I_{c}=0.13 / \$ /$ year, and $K=500$ units.

Then the optimal solutions are $\left\{Z_{11}=\$ 7404.27, T^{*}=\right.$ 0.70 year, $t_{1}^{*}=0.17$ year $\},\left\{Z_{12}=\$ 6895.63, T^{*}=0.62\right.$ year, $t_{1}^{*}=0.14$ year $\},\left\{Z_{13}=\$ 6689.02, T^{*}=0.58\right.$ year, $t_{1}^{*}=0.13$ year $\},\left\{Z_{21}=\$ 7149.18, T^{*}=0.33\right.$ year, $t_{1}^{*}=0.07$ year $\}$, $\left\{Z_{22}=\$ 6816.19, T^{*}=0.41\right.$ year, $t_{1}^{*}=0.09$ year $\}$, and $\left\{Z_{23}=\right.$ $\$ 6677, T^{*}=0.49$ year, $t_{1}^{*}=0.11$ year $\}$.

Among the above optimal solutions, the optimal solution is $\left\{Z_{11}=\$ 7404.27, T^{*}=0.70\right.$ year, $t_{1}^{*}=0.17$ year $\}$ global maximum. The average profit function is highly nonlinear. Figure 4 shows the concavity of the functions and comparisons of the cost functions.

Example C. We consider the following parametric values in appropriate units: $C_{1}=\$ 250$ per order, $C_{2}=\$ 20 /$ unit/year, $R_{1}=120 / 365$ year, $R_{2}=150 / 365$ year, $R_{3}=180 / 365$ year, $\rho_{1}=10 \%, \rho_{2}=5 \%, \rho_{3}=2 \%, a=100$ units, $D(t)=a, C_{4}=$ $\$ 120 /$ unit, $P=\$ 180 /$ unit, $I_{f}=0.16 / \$ /$ year, $I_{c}=0.13 / \$ /$ year, and $K=500$.

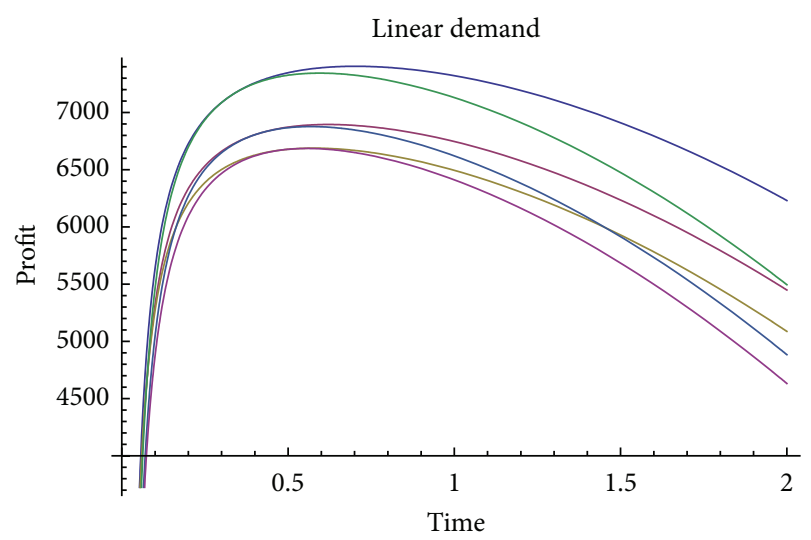

FIgURE 4: The figures of the average profit functions are in descending order as $Z_{11}, Z_{21}, Z_{12}, Z_{22}, Z_{13}$, and $Z_{23}$.

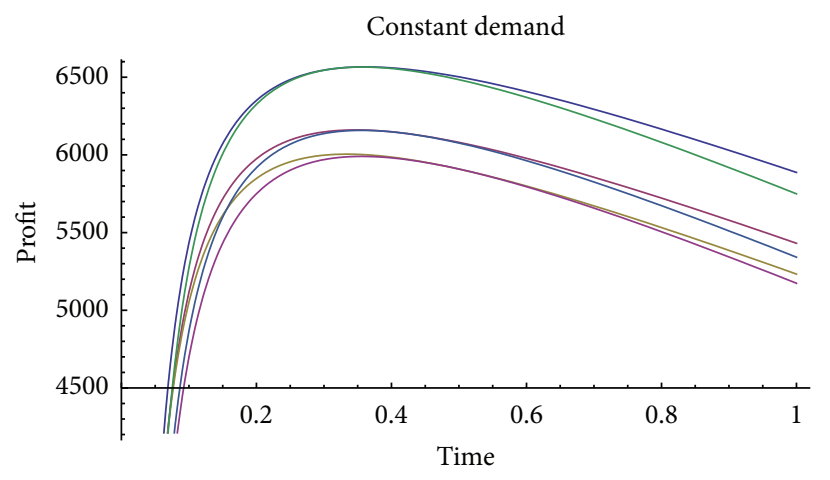

FIgURE 5: The figures of the average profit functions are in descending order as $Z_{11}, Z_{21}, Z_{12}, Z_{22}, Z_{13}$, and $Z_{23}$.

Then the optimal solutions are $\left\{Z_{11}=\$ 6566.5, T^{*}=\right.$ 0.36 year, $t_{1}^{*}=0.07$ year $\},\left\{Z_{12}=\$ 6160.63, T^{*}=0.35\right.$ year, $t_{1}^{*}=0.07$ year $\},\left\{Z_{13}=\$ 6004.87, T^{*}=0.33\right.$ year, $t_{1}^{*}=0.07$ year $\},\left\{Z_{21}=\$ 6561.20, T^{*}=0.33\right.$ year, $t_{1}^{*}=0.07$ year $\}$, $\left\{Z_{22}=\$ 6143.73, T^{*}=0.41\right.$ year, $t_{1}^{*}=0.08$ year $\}$, and $\left\{Z_{23}=\right.$ $\$ 5915.57, T^{*}=0.49$ year, $t_{1}^{*}=0.10$ year $\}$.

From the above optimal solutions, the optimal solution is $\left\{Z_{11}=\$ 6566.5, T^{*}=0.36\right.$ year, $t_{1}^{*}=0.07$ year $\}$ global maximum. We obtain a closed type formula for $T^{*}$, and Figure 5 shows the concavity of the functions and comparisons of the cost functions.

Example D. We consider the following parametric values in appropriate units: $C_{1}=\$ 250$ per order, $C_{2}=\$ 10 /$ unit $/$ month, $R_{1}=1$ month, $R_{2}=2$ months, $R_{3}=3$ months, $\rho_{1}=$ $20 \%, \rho_{2}=15 \%, \rho_{3}=10 \%, a=50$ units, $b=0.16$ units, $D(t)=a \exp (b t), C_{4}=\$ 200 /$ unit, $P=\$ 265 /$ unit, $I_{f}=$ $0.16 \$ /$ month, $I_{c}=0.13 \$ / \mathrm{month}$, and $K=500$ units.

Then the optimal solutions are $\left\{Z_{11}=\$ 5840.84, T^{*}=\right.$ 5.86 months, $t_{1}^{*}=0.97$ month $\},\left\{Z_{12}=\$ 5227.55, T^{*}=\right.$ 4.51 months, $t_{1}^{*}=0.66$ month $\},\left\{Z_{13}=\$ 4712.47, T^{*}=\right.$ 3.32 months, $t_{1}^{*}=0.44$ month $\},\left\{Z_{21}=\$ 5270.15, T^{*}=1\right.$ month, $t_{1}^{*}=0.11$ month $\},\left\{Z_{22}=\$ 5081.96, T^{*}=2\right.$ months, 


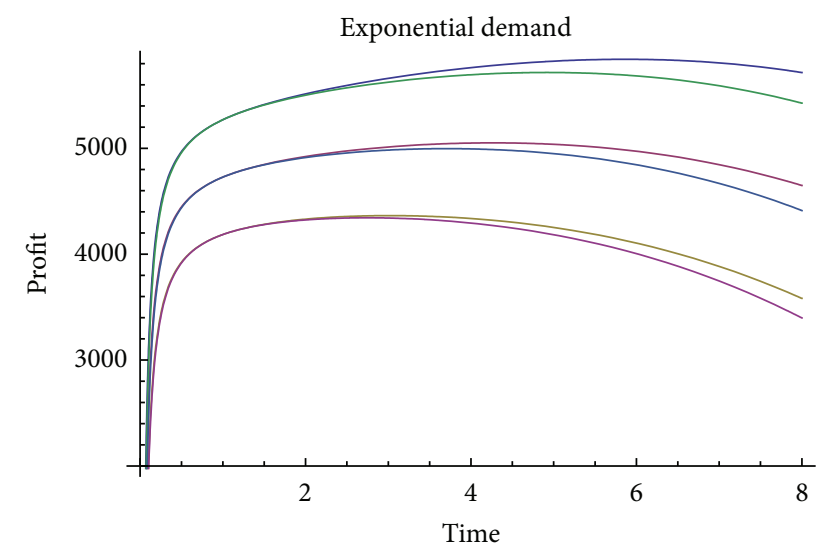

FIGURE 6: The figures of the average profit functions are in descending order as $Z_{11}, Z_{21}, Z_{12}, Z_{22}, Z_{13}$, and $Z_{23}$.

$t_{1}^{*}=0.24$ month $\}$, and $\left\{Z_{23}=\$ 4710.04, T^{*}=3\right.$ months, $t_{1}^{*}=$ 0.39 month\}.

From the above optimal solutions, the optimal solution is $\left\{Z_{11}=\$ 5840.84, T^{*}=5.86\right.$ months, $t_{1}^{*}=0.97$ month $\}$ global maximum. The average profit function is highly non-linear. Figure 6 shows the concavity of the functions and comparisons of the cost functions.

Sensitivity Analysis. The sensitivity analysis of the key parameters is given in Table 2 for quadratic demand, Table 3 for linear demand, Table 4 for constant demand, and Table 5 for exponential demand.

(i) If ordering cost increases, then material handlling cost, shipping cost, and placing order's cost increase; as a result the total relevant profit decreases.

(ii) If the unit holding cost per unit item increases, the total profit of the system decreases.

(iii) Increasing value of maximum retailer price increases the total purchasing cost of the whole system which decreases the total profit.

(iv) Increasing value of selling price per item increases the total selling price per lot which reduces the total cost of the whole system. Therefore, the total profit of the system increases.

(v) If we increase the replenishment rate then the holding cost increases which results the decreasing value of the total profit.

\section{Concluding Remarks}

In most EOQ models, the retailer pays the purchasing cost of items to the supplier as soon as the items are received. In the competitive marketing environment, the supplier offers the retailer a delay period called the trade credit period to stimulate the retailer to buy more items. Before the end of the trade credit period, the retailer can sell his products, accumulate revenue, and earn interest. Besides it, a higher interest is
TABLE 2: Sensitivity analysis for the quadratic demand pattern.

\begin{tabular}{|c|c|c|c|c|}
\hline Parameters & $\%$ & $Z_{1}$ & $Z_{2}$ & $Z_{3}$ \\
\hline \multicolumn{5}{|c|}{ Quadratic demand Case 1} \\
\hline \multirow{4}{*}{$C_{0}$} & $-50 \%$ & 2.14 & 2.80 & 3.24 \\
\hline & $-25 \%$ & 1.02 & 1.31 & 1.49 \\
\hline & $+25 \%$ & -0.95 & -1.20 & -1.35 \\
\hline & $+50 \%$ & -1.86 & -2.32 & -2.59 \\
\hline \multirow{4}{*}{$C_{H}$} & $-50 \%$ & 8.17 & 6.89 & 6.27 \\
\hline & $-25 \%$ & 3.34 & 2.89 & 2.66 \\
\hline & $+25 \%$ & -2.50 & -2.25 & -2.11 \\
\hline & $+50 \%$ & -4.50 & -4.11 & -3.87 \\
\hline \multirow{4}{*}{$C_{R}$} & $-50 \%$ & 18.02 & 25.83 & 29.23 \\
\hline & $-25 \%$ & 55.72 & 58.33 & 59.46 \\
\hline & $+25 \%$ & -42.46 & -47.02 & -49.56 \\
\hline & $+50 \%$ & -82.40 & -92.16 & -97.71 \\
\hline \multirow{4}{*}{$P$} & $-50 \%$ & -137.21 & -148.03 & -154.16 \\
\hline & $-25 \%$ & -70.15 & -75.12 & -77.87 \\
\hline & $+25 \%$ & 78.03 & 166.01 & 221.24 \\
\hline & $+50 \%$ & 54.07 & 223.77 & 221.87 \\
\hline \multirow{4}{*}{$K$} & $-50 \%$ & 4.76 & 3.76 & 3.34 \\
\hline & $-25 \%$ & 1.24 & 1.05 & 0.95 \\
\hline & $+25 \%$ & -0.66 & -0.57 & 0.52 \\
\hline & $+50 \%$ & -1.06 & -0.92 & 0.85 \\
\hline \multicolumn{5}{|c|}{ Quadratic demand Case 2} \\
\hline \multirow{4}{*}{$C_{0}$} & $-50 \%$ & 5.30 & 4.44 & 3.77 \\
\hline & $-25 \%$ & 2.65 & 2.22 & 1.88 \\
\hline & $+25 \%$ & -2.65 & -2.22 & -1.88 \\
\hline & $+50 \%$ & -5.30 & -4.44 & -3.77 \\
\hline \multirow{4}{*}{$C_{H}$} & $-50 \%$ & 2.01 & 2.70 & 3.37 \\
\hline & $-25 \%$ & 1.01 & 1.35 & 1.69 \\
\hline & $+25 \%$ & -1.01 & -1.35 & -1.69 \\
\hline & $+50 \%$ & -2.01 & -2.70 & -3.37 \\
\hline \multirow{4}{*}{$C_{R}$} & $-50 \%$ & 81.72 & 92.20 & 98.91 \\
\hline & $-25 \%$ & 40.86 & 46.00 & 49.45 \\
\hline & $+25 \%$ & -40.86 & -46.00 & -49.45 \\
\hline & $+50 \%$ & -81.72 & -92.20 & -98.91 \\
\hline \multirow{4}{*}{$P$} & $-50 \%$ & -139.04 & -149.34 & -156.05 \\
\hline & $-25 \%$ & -69.52 & -74.67 & -78.03 \\
\hline & $+25 \%$ & 69.52 & 74.67 & 78.03 \\
\hline & $+50 \%$ & 139.04 & 149.34 & 156.05 \\
\hline \multirow{4}{*}{ K } & $-50 \%$ & 1.08 & 1.47 & 1.88 \\
\hline & $-25 \%$ & 0.36 & 0.49 & 0.62 \\
\hline & $+25 \%$ & -0.22 & -0.29 & -0.37 \\
\hline & $+50 \%$ & -0.36 & -0.49 & -0.62 \\
\hline
\end{tabular}

charged if the payment is not settled by the end of the trade credit period. Several papers discussing the topic have been mentioned in the literature that investigates inventory problems under various conditions. Many articles in this direction consider the replenishment rate to be instantaneously infinite, but its size is finite and the lot is placed in the beginning of the cycle. Some models consider finite replenishment/supply rate for constant demand rate. Our models 
TABLE 3: Sensitivity analysis for the linear demand pattern.

\begin{tabular}{|c|c|c|c|c|}
\hline Parameters & $\%$ & $Z_{1}$ & $Z_{2}$ & $Z_{3}$ \\
\hline \multicolumn{5}{|c|}{ Linear demand Case 1} \\
\hline \multirow{4}{*}{$C_{0}$} & $-50 \%$ & 2.67 & 3.32 & 3.75 \\
\hline & $-25 \%$ & 1.26 & 1.54 & 1.72 \\
\hline & $+25 \%$ & -1.16 & -1.40 & -1.54 \\
\hline & $+50 \%$ & -2.24 & -2.69 & -2.96 \\
\hline \multirow{4}{*}{$C_{H}$} & $-50 \%$ & 5.84 & 5.29 & 4.97 \\
\hline & $-25 \%$ & 2.54 & 2.34 & 2.21 \\
\hline & $+25 \%$ & -2.07 & -1.95 & -1.86 \\
\hline & $+50 \%$ & -3.82 & -3.63 & -3.47 \\
\hline \multirow{4}{*}{$C_{R}$} & $-50 \%$ & 112.63 & 121.07 & 124.66 \\
\hline & $-25 \%$ & 47.64 & 51.69 & 53.57 \\
\hline & $+25 \%$ & -41.94 & -46.70 & -49.32 \\
\hline & $+50 \%$ & -82.21 & -92.11 & -97.72 \\
\hline \multirow{4}{*}{$P$} & $-50 \%$ & -137.74 & -148.48 & -154.55 \\
\hline & $-25 \%$ & -69.87 & -74.97 & -77.78 \\
\hline & $+25 \%$ & 77.20 & 80.96 & 82.68 \\
\hline & $+50 \%$ & 169.90 & 176.81 & 179.62 \\
\hline \multirow{4}{*}{ K } & $-50 \%$ & 3.14 & 2.80 & 2.60 \\
\hline & $-25 \%$ & 0.92 & 0.84 & 0.78 \\
\hline & $+25 \%$ & -0.51 & -0.47 & -0.44 \\
\hline & $+50 \%$ & -0.83 & -0.77 & -0.73 \\
\hline \multicolumn{5}{|c|}{ Linear demand Case 2} \\
\hline \multirow{4}{*}{$C_{0}$} & $-50 \%$ & 5.32 & 4.46 & 3.80 \\
\hline & $-25 \%$ & 2.66 & 2.23 & 1.90 \\
\hline & $+25 \%$ & -2.66 & -2.23 & -1.90 \\
\hline & $+50 \%$ & -5.32 & -4.46 & -3.80 \\
\hline \multirow{4}{*}{$C_{H}$} & $-50 \%$ & 2.38 & 3.24 & 4.13 \\
\hline & $-25 \%$ & 1.01 & 1.35 & 1.68 \\
\hline & $+25 \%$ & -1.01 & -1.35 & -1.68 \\
\hline & $+50 \%$ & -2.01 & -2.69 & -3.37 \\
\hline \multirow{4}{*}{$C_{R}$} & $-50 \%$ & 81.74 & 92.22 & 98.92 \\
\hline & $-25 \%$ & 40.87 & 46.11 & 49.46 \\
\hline & $+25 \%$ & -40.87 & -46.11 & -49.46 \\
\hline & $+50 \%$ & -81.74 & -92.22 & -98.92 \\
\hline \multirow{4}{*}{$P$} & $-50 \%$ & -139.07 & -149.37 & -78.08 \\
\hline & $-25 \%$ & -69.54 & -74.69 & -78.04 \\
\hline & $+25 \%$ & 69.54 & 74.69 & 78.04 \\
\hline & $+50 \%$ & 139.07 & 149.37 & 156.08 \\
\hline \multirow{4}{*}{ K } & $-50 \%$ & 1.08 & 1.47 & 1.86 \\
\hline & $-25 \%$ & 0.36 & 0.49 & 0.62 \\
\hline & $+25 \%$ & -0.22 & -0.29 & -0.37 \\
\hline & $+50 \%$ & 1.08 & 1.47 & 1.86 \\
\hline
\end{tabular}

consider the demand of the products as an increasing function of time. From the view point of the supplier, price discount on the purchasing cost of the items by retailer is given at a different delay period to motivate the retailer to buy more. Generally, suppliers consider maximum delay period, after which they would not take any risk of getting back money from retailers. As a result, the purchasing cost $C_{3}$ is infinite when $R$ is greater than $R_{3}$; that is, retailer never purchases
TABLE 4: Sensitivity analysis for the constant demand pattern.

\begin{tabular}{|c|c|c|c|c|}
\hline Parameters & $\%$ & $Z_{1}$ & $Z_{2}$ & $Z_{3}$ \\
\hline \multicolumn{5}{|c|}{ Constant demand Case 1} \\
\hline \multirow{4}{*}{$C_{0}$} & $-50 \%$ & 6.39 & 7.18 & 7.80 \\
\hline & $-25 \%$ & 2.86 & 3.18 & 3.42 \\
\hline & $+25 \%$ & -2.74 & -2.73 & -2.90 \\
\hline & $+50 \%$ & -4.68 & -5.15 & -5.46 \\
\hline \multirow{4}{*}{$C_{H}$} & $-50 \%$ & 2.35 & 2.40 & 2.37 \\
\hline & $-25 \%$ & 1.13 & 1.16 & 3.79 \\
\hline & $+25 \%$ & -1.07 & -1.10 & -3.60 \\
\hline & $+50 \%$ & -2.08 & -2.14 & -4.60 \\
\hline \multirow{4}{*}{$C_{R}$} & $-50 \%$ & 82.26 & 92.65 & 93.61 \\
\hline & $-25 \%$ & 41.13 & 46.31 & 45.47 \\
\hline & $+25 \%$ & -41.13 & -46.30 & -50.49 \\
\hline & $+50 \%$ & -82.25 & -92.59 & -98.38 \\
\hline \multirow{4}{*}{$P$} & $-50 \%$ & -139.56 & -150.26 & -156.00 \\
\hline & $-25 \%$ & -69.82 & -75.23 & -78.76 \\
\hline & $+25 \%$ & 69.92 & 75.51 & 74.24 \\
\hline & $+50 \%$ & 139.96 & 151.45 & 159.23 \\
\hline \multirow{4}{*}{$K$} & $-50 \%$ & 1.13 & 1.16 & 1.15 \\
\hline & $-25 \%$ & 0.37 & 0.38 & 0.37 \\
\hline & $+25 \%$ & -0.22 & -0.22 & -0.22 \\
\hline & $+50 \%$ & -0.36 & -0.37 & -0.37 \\
\hline \multicolumn{5}{|c|}{ Constant demand Case 2} \\
\hline \multirow{4}{*}{$C_{0}$} & $-50 \%$ & 5.80 & 4.95 & 4.28 \\
\hline & $-25 \%$ & 2.90 & 2.48 & 2.14 \\
\hline & $+25 \%$ & -2.90 & -2.48 & -2.14 \\
\hline & $+50 \%$ & -5.80 & -4.95 & -4.28 \\
\hline \multirow{4}{*}{$C_{H}$} & $-50 \%$ & 2.00 & 2.68 & 3.33 \\
\hline & $-25 \%$ & 1.00 & 1.34 & 1.67 \\
\hline & $+25 \%$ & -1.00 & -1.34 & -1.67 \\
\hline & $+50 \%$ & -2.00 & -2.68 & -3.33 \\
\hline \multirow{4}{*}{$C_{R}$} & $-50 \%$ & 82.30 & 92.78 & 99.40 \\
\hline & $-25 \%$ & 41.15 & 46.39 & 49.70 \\
\hline & $+25 \%$ & -41.15 & -46.39 & -49.70 \\
\hline & $+50 \%$ & 82.30 & -92.78 & -99.40 \\
\hline \multirow{4}{*}{$P$} & $-50 \%$ & -140.10 & -150.40 & -157.02 \\
\hline & $-25 \%$ & -70.05 & -75.20 & -78.51 \\
\hline & $+25 \%$ & 70.05 & 75.20 & 78.51 \\
\hline & $+50 \%$ & 140.10 & 150.40 & 157.02 \\
\hline \multirow{4}{*}{ K } & $-50 \%$ & 1.00 & 1.34 & 1.67 \\
\hline & $-25 \%$ & 0.33 & 0.44 & 0.56 \\
\hline & $+25 \%$ & -0.20 & -0.27 & -0.33 \\
\hline & $+50 \%$ & -0.33 & -0.44 & -0.56 \\
\hline
\end{tabular}

items at infinite cost. In addition, we establish eight effective and easy-to-use lemmas and figures to help the retailer to take optimal strategy for his marketing policy. We solved the model analytically. We found global optimal solutions for different types of demand patterns. We illustrate the numerical results graphically. As far as the knowledge of authors goes, such type of model has not yet been discussed in the EOQ literature. The application of our model in a different 
TABLE 5: Sensitivity analysis for the exponential demand pattern.

\begin{tabular}{|c|c|c|c|c|}
\hline Parameters & $\%$ & $Z_{1}$ & $Z_{2}$ & $Z_{3}$ \\
\hline \multicolumn{5}{|c|}{ Exponential demand Case 1} \\
\hline \multirow{4}{*}{$C_{0}$} & $-50 \%$ & 0.37 & 0.54 & 0.83 \\
\hline & $-25 \%$ & 0.18 & -0.27 & 0.41 \\
\hline & $+25 \%$ & -0.18 & -0.26 & -0.39 \\
\hline & $+50 \%$ & -0.36 & -0.52 & -0.77 \\
\hline \multirow{4}{*}{$C_{H}$} & $-50 \%$ & - & - & - \\
\hline & $-25 \%$ & 18.16 & 13.01 & 9.39 \\
\hline & $+25 \%$ & -6.93 & -5.23 & -3.78 \\
\hline & $+50 \%$ & -10.30 & -7.89 & -5.80 \\
\hline \multirow{4}{*}{$C_{R}$} & $-50 \%$ & - & - & - \\
\hline & $-25 \%$ & - & - & - \\
\hline & $+25 \%$ & -46.01 & -46.01 & -50.15 \\
\hline & $+50 \%$ & -84.03 & -84.03 & -95.06 \\
\hline \multirow{4}{*}{$P$} & $-50 \%$ & - & - & - \\
\hline & $-25 \%$ & -71.44 & -76.39 & -83.42 \\
\hline & $+25 \%$ & - & - & - \\
\hline & $+50 \%$ & - & - & - \\
\hline \multirow{4}{*}{ K } & $-50 \%$ & - & - & 5.03 \\
\hline & $-25 \%$ & 2.82 & 1.80 & 1.16 \\
\hline & $+25 \%$ & -1.25 & -0.86 & -0.57 \\
\hline & $+50 \%$ & -1.97 & -1.37 & -0.92 \\
\hline \multicolumn{5}{|c|}{ Exponential demand Case 2} \\
\hline \multirow{4}{*}{$C_{0}$} & $-50 \%$ & 2.37 & 1.23 & 0.88 \\
\hline & $-25 \%$ & 1.86 & 0.61 & 0.44 \\
\hline & $+25 \%$ & -1.18 & -0.61 & -0.44 \\
\hline & $+50 \%$ & -2.37 & -1.23 & -0.88 \\
\hline \multirow{4}{*}{$C_{H}$} & $-50 \%$ & 2.36 & 5.42 & 9.72 \\
\hline & $-25 \%$ & 1.18 & 2.71 & 4.86 \\
\hline & $+25 \%$ & -1.18 & -2.71 & -4.86 \\
\hline & $+50 \%$ & -2.36 & -2.36 & -9.72 \\
\hline \multirow{4}{*}{$C_{R}$} & $-50 \%$ & 82.31 & 98.56 & 122.63 \\
\hline & $-25 \%$ & 41.15 & 49.28 & 61.31 \\
\hline & $+25 \%$ & -41.15 & -49.28 & -61.31 \\
\hline & $+50 \%$ & -82.31 & -98.56 & - \\
\hline \multirow{4}{*}{$P$} & $-50 \%$ & - & - & - \\
\hline & $-25 \%$ & -68.52 & -77.61 & -91.62 \\
\hline & $+25 \%$ & 68.52 & 77.61 & 91.62 \\
\hline & $+50 \%$ & 137.04 & 155.21 & 183.23 \\
\hline \multirow{4}{*}{ K } & $-50 \%$ & 0.56 & 1.37 & 2.62 \\
\hline & $-25 \%$ & 0.18 & 0.46 & 0.87 \\
\hline & $+25 \%$ & -0.11 & -0.27 & -0.52 \\
\hline & $+50 \%$ & -0.18 & -0.46 & -0.87 \\
\hline
\end{tabular}

- indicates the value of the parameter is either imaginary or the value does not exist at that point.

field of inventory models where the assumptions are valid. Further extension of the model may be generalized by considering shortages, deterioration, and stochastic demand. One immediate extension of this model is to consider partial backlogging.

\section{Appendices}

\section{A. Derivation of Lemmas 1 and 2}

Differentiating (13), the equations become

$$
\begin{gathered}
\frac{d Z_{1 i}}{d T}=\left[\left(P-C_{3}\right)\left(\frac{b}{2}+\frac{2 c T}{3}\right)-\frac{I_{c} P}{T^{2}}\left\{\frac{a R_{i}^{2}}{2}+\frac{b R_{i}^{3}}{6}+\frac{c R_{i}^{4}}{12}\right\}\right. \\
-C_{2}\left\{\frac { 1 } { 2 K } \left(a^{2}+\frac{3 b^{2} T^{2}}{4}+\frac{5 c^{2} T^{4}}{9}+2 a b T\right.\right. \\
\left.+\frac{4 b c T^{3}}{3}+2 a c T^{2}\right)+\frac{a}{2}+\frac{2 b T}{3} \\
+\frac{3 c T^{2}}{4}-\frac{a^{2}}{K}-\frac{2 a b T}{K}-\frac{2 a c T^{2}}{K} \\
\left.-\frac{3 b^{2} T^{2}}{4 K}-\frac{4 b c T^{3}}{3 K}-\frac{5 c^{2} T^{4}}{9 K}\right\} \\
-I_{f} C_{3}\left\{\frac{a}{2}+\frac{2 b T}{3}+\frac{3 c T^{2}}{4}-\frac{a R_{i}^{2}}{2 T^{2}}-\frac{b R_{i}}{2}-\frac{b R_{i}^{3}}{6 T^{2}}\right. \\
\left.\left.-\frac{2 c T R_{i}}{3}-\frac{c R_{i}^{4}}{12 T^{2}}\right\}+\frac{C_{1}}{T^{2}}\right] \\
=\frac{1}{T^{2}}\left[\xi_{1}(c) T^{6}+\xi_{2}(b, c) T^{5}+\xi_{3}(a, b, c) T^{4}\right. \\
\left.+\xi_{4}(a, b, c) T^{3}+\xi_{5}(a, b) T^{2}+\xi_{6}(a, b, c)\right]
\end{gathered}
$$

where

$$
\begin{gathered}
\xi_{1}(c)=\frac{5 c^{2} C_{2}}{18 K}, \quad \xi_{2}(b, c)=\frac{2 b c C_{2}}{3 K}, \\
\xi_{3}(a, b, c)=\left\{\left(2 a c+\frac{3 b^{2}}{4}\right) \frac{C_{2}}{2 K}-\left(C_{2}+I_{f} C_{3}\right) \frac{3 c}{4}\right\}, \\
\xi_{4}(a, b, c)=\left\{\left(P-C_{3}\right) \frac{2 c}{3}+\frac{C_{2} a b}{K}\right. \\
\left.-\left(C_{2}+I_{f} C_{3}\right) \frac{2 b}{3}+\frac{2 I_{f} C_{3} c R_{i}}{3}\right\}, \\
\xi_{5}(a, b)=\left\{\left(P-C_{3}\right) \frac{b}{2}+\frac{C_{2} a^{2}}{K}\right. \\
\left.-\left(C_{2}+I_{f} C_{3}\right) \frac{a}{2}+\frac{I_{f} C_{3} b R_{i}}{2}\right\}, \\
\xi_{6}(a, b, c)=C_{1}+\left\{\frac{a R_{i}^{2}}{2}+\frac{b R_{i}^{3}}{6}+\frac{c R_{i}^{4}}{12}\right\}\left(I_{f} C_{3}-I_{c} P\right),
\end{gathered}
$$




$$
\begin{array}{r}
\frac{d^{2} Z_{1 i}}{d T^{2}}=\left[\left(P-C_{3}\right)\left(\frac{2 c}{3}\right)+\frac{2 I_{c} P}{T^{3}}\left\{\frac{a R_{i}^{2}}{2}+\frac{b R_{i}^{3}}{6}+\frac{c R_{i}^{4}}{12}\right\}\right. \\
-C_{2}\left\{\frac { 1 } { 2 K } \left(\frac{3 b^{2} T}{2}+\frac{20 c^{2} T^{3}}{9}+2 a b+4 b c T^{2}\right.\right. \\
+4 a c T)+\frac{2 b}{3}+\frac{3 c T}{2}-\frac{2 a b}{K} \\
\left.-\frac{4 a c T}{K}-\frac{3 b^{2} T}{2 K}-\frac{4 b c T^{2}}{K}-\frac{20 c^{2} T^{3}}{9 K}\right\} \\
-I_{f} C_{3}\left\{\frac{2 b}{3}+\frac{3 c T}{2}+\frac{a R_{i}^{2}}{T^{3}}+\frac{b R_{i}^{3}}{3 T^{3}}\right. \\
\left.\left.-\frac{2 c R_{i}}{3}+\frac{c R_{i}^{4}}{6 T^{3}}\right\}-\frac{2 C_{1}}{T^{3}}\right] \\
=\frac{1}{T^{3}}\left[4 \xi_{1}(c) T^{6}+\xi_{2}(b, c) T^{5}+\xi_{3}(a, b, c) T^{4}\right. \\
\left.+\xi_{4}(a, b, c) T^{3}-2 \xi_{6}(a, b, c)\right] .
\end{array}
$$

Now,

$$
\begin{gathered}
\frac{d Z_{2 i}}{d T}=\left[\left(P-C_{3}\right)\left(\frac{b}{2}+\frac{2 c T}{3}\right)\right. \\
-I_{c} P\left\{\frac{2 c T R_{i}}{3}+\frac{b R_{i}}{2}-\frac{a}{2}-\frac{2 b T}{3}-\frac{3 c T^{2}}{4}\right\} \\
-C_{2}\left\{\frac { 1 } { 2 K } \left(a^{2}+\frac{3 b^{2} T^{2}}{4}+\frac{5 c^{2} T^{4}}{9}\right.\right. \\
\left.+2 a b T+\frac{4 b c T^{3}}{3}+2 a c T^{2}\right)+\frac{a}{2}+\frac{2 b T}{3} \\
+\frac{3 c T^{2}}{4}-\frac{a^{2}}{K}-\frac{2 a b T}{K}-\frac{2 a c T^{2}}{K} \\
\left.\left.-\frac{3 b^{2} T^{2}}{4 K}-\frac{4 b c T^{3}}{3 K}-\frac{5 c^{2} T^{4}}{9 K}\right\}+\frac{C_{1}}{T^{2}}\right] \\
=\frac{1}{T^{2}}\left[\xi_{1}(c) T^{6}+\xi_{2}(b, c) T^{5}+\xi_{7}(a, b, c) T^{4}\right. \\
\left.+\xi_{8}(a, b, c) T^{3}+\xi_{9}(a, b, c) T^{2}+C_{1}\right],
\end{gathered}
$$

where

$$
\begin{gathered}
\xi_{1}(c)=\frac{5 c^{2} C_{2}}{18 K}, \quad \xi_{2}(b, c)=\frac{2 b c C_{2}}{3 K}, \\
\xi_{7}(a, b, c)=\left\{\left(2 a c+\frac{3 b^{2}}{4}\right) \frac{C_{2}}{2 K}-\left(C_{2}+I_{c} P\right) \frac{3 c}{4}\right\},
\end{gathered}
$$

$$
\begin{gathered}
\xi_{8}(a, b, c)=\left\{\left(P-C_{3}\right) \frac{2 c}{3}+\frac{C_{2} a b}{K}\right. \\
\left.-\left(C_{2}+I_{c} P\right) \frac{2 b}{3}+\frac{2 I_{c} P c R_{i}}{3}\right\}, \\
\xi_{9}(a, b)=\left\{\left(P-C_{3}\right) \frac{b}{2}+\frac{C_{2} a^{2}}{K}-\left(C_{2}+I_{c} P\right) \frac{a}{2}+\frac{I_{c} P b R_{i}}{2}\right\}, \\
\frac{d^{2} Z_{2 i}}{d T^{2}}=\left[\left(P-C_{3}\right)\left(\frac{2 c}{3}\right)-I_{c} P\left\{\frac{2 c R_{i}}{3}-\frac{2 b}{3}-\frac{3 c T}{2}\right\}\right. \\
-C_{2}\left\{\frac { 1 } { 2 K } \left(\frac{3 b^{2} T}{2}+\frac{20 c^{2} T^{3}}{9}\right.\right. \\
\left.+2 a b+4 b c T^{2}+4 a c T\right) \\
+\frac{2 b}{3}+\frac{3 c T}{2}-\frac{2 a b}{K}-\frac{4 a c T}{K} \\
\left.\left.-\frac{3 b^{2} T}{2 K}-\frac{4 b c T^{2}}{K}-\frac{20 c^{2} T^{3}}{9 K}\right\}-\frac{2 C_{1}}{T^{3}}\right] \\
=\frac{1}{T^{3}}\left[5 \xi_{1}(c) T^{6}+\xi_{2}(b, c) T^{5}\right. \\
\left.+2 \xi_{7}(a, b, c) T^{4}+\xi_{8}(a, b, c) T^{3}-2 C_{1}\right] .
\end{gathered}
$$

To obtain the optimal cycle length, proof of two lemmas are as follows.

Proof of Lemma 1. The first part of the lemma is obvious from the above calculations of $d Z_{1 i} / d T$ and $d^{2} Z_{1 i} / d T^{2}$. For the second part, if $Z_{1 i}$ does not have any stationary points in $\left[R_{i}, \infty\right)$, then $Z_{1 i}$ is either monotonic increasing or monotonic decreasing function of $T \in\left[R_{i}, \infty\right)$. Here, $d Z_{1 i} / d T \rightarrow$ $\infty$ as $T \rightarrow \infty$ because $\xi_{1}(c)>0$. Hence, $Z_{1 i}$ will be monotonic increasing if $\left.\left(d Z_{1 i} / d T\right)\right|_{T=R_{i}}>0$ as $Z_{1 i}$ does not have stationary points in $\left[R_{i}, \infty\right)$. Here, $t_{1}=(1 / K)(a T+$ $\left.(b / 2) T^{2}+(c / 3) T^{3}\right), t_{1}$ increase with increasing value of $T$. As in our models $t_{1} \leq R_{i}$; hence, for the feasibility of the model, $\left(t_{1}\right)^{*}=R_{i}$ and $T^{*}$ is obtained from $(c / 3)\left(T^{*}\right)^{3}+(b / 2)\left(T^{*}\right)^{2}+$ $a\left(T^{*}\right)=K R_{i}$. By Descarte's rule, $(c / 3)\left(T^{*}\right)^{3}+(b / 2)\left(T^{*}\right)^{2}+$ $a\left(T^{*}\right)-K R_{i}=0$ may have atmost one positive root and two negative roots. Therefore, $Z_{1 i}$ has a maximum value at $T^{*}=$ $\left\{T \mid(c / 3) T^{3}+(b / 2) T^{2}+a T-K R_{i}=0, T>0\right\}$ if $\left(1 / R_{i}^{2}\right)\left[\xi_{1}(c) R_{i}^{6}+\xi_{2}(b, c) R_{i}^{5}+\xi_{3}(a, b, c) R_{i}^{4}+\xi_{4}(a, b, c) R_{i}^{3}+\right.$ $\left.\xi_{5}(a, b) R_{i}^{2}+\xi_{6}(a, b, c)\right]>0$. Here $T^{*}$ is unique if it exists, by Descarte's rule, hence the proof.

Proof of Lemma 2. The first part of the lemma is obvious (see the expressions of $d Z_{1 i} / d T$ and $d^{2} Z_{1 i} / d T^{2}$ ). For the second part, if $Z_{2 i}$ does not have any stationary points in $\left[0, R_{i}\right]$, then $Z_{2 i}$ is either monotonic increasing or monotonic decreasing function of $T \in\left[0, R_{i}\right]$. Here $d Z_{2 i} / d T \rightarrow \infty$ as $T \rightarrow$ 0 because $C_{1}>0$. Hence, $Z_{2 i}$ is monotonic increasing if $\left.\left(d Z_{2 i} / d T\right)\right|_{T=R_{i}}>0$ as $Z_{2 i}$ does not have stationary points in $\left[0, R_{i}\right]$. Hence $Z_{2 i}$ has a maximum value at $T=R_{i}$ if 
$\left[\left(R_{i}\right)^{6} \xi_{1}(c)+\left(R_{i}\right)^{5} \xi_{2}(b, c)+\left(R_{i}\right)^{4} \xi_{7}(a, b, c)+\left(R_{i}\right)^{3} \xi_{8}(a, b, c)+\right.$ $\left.\left(R_{i}\right)^{2} \xi_{9}(a, b, c)+C_{1}\right]>0$, hence the proof.

\section{B. Derivation of Lemmas 3 and 4}

Now differentiating (16), we obtain

$$
\begin{aligned}
\frac{d Z_{1 i}}{d T}=\left[\left(P-C_{3}\right)\left(\frac{b}{2}\right)-\frac{I_{c} P}{T^{2}}\left\{\frac{a R_{i}^{2}}{2}+\frac{b R_{i}^{3}}{6}\right\}\right. & -C_{2}\left\{\frac{1}{2 K}\left(a^{2}+\frac{3 b^{2} T^{2}}{4}+2 a b T\right)+\frac{a}{2}\right. \\
& \left.+\frac{2 b T}{3}-\frac{a^{2}}{K}-\frac{2 a b T}{K}-\frac{3 b^{2} T^{2}}{4 K}\right\} \\
& \left.-I_{f} C_{3}\left\{\frac{a}{2}+\frac{2 b T}{3}-\frac{a R_{i}^{2}}{2 T^{2}}-\frac{b R_{i}}{2}-\frac{b R_{i}^{3}}{6 T^{2}}\right\}+\frac{C_{1}}{T^{2}}\right] \\
= & \frac{1}{T^{2}}\left[\xi_{3}(a, b, 0) T^{4}+\xi_{4}(a, b, 0) T^{3}\right. \\
& \left.+\xi_{5}(a, b) T^{2}+\xi_{6}(a, b, 0)\right]
\end{aligned}
$$

where

$$
\begin{gathered}
\xi_{3}(a, b, 0)=\left(\frac{3 b^{2}}{4}\right) \frac{C_{2}}{2 K}, \\
\xi_{4}(a, b, 0)=\left\{\frac{C_{2} a b}{K}-\left(C_{2}+I_{f} C_{3}\right) \frac{2 b}{3}\right\},
\end{gathered}
$$

$\xi_{5}(a, b)$

$$
\begin{gathered}
=\left\{\left(P-C_{3}\right) \frac{b}{2}+\frac{C_{2} a^{2}}{K}-\left(C_{2}+I_{f} C_{3}\right) \frac{a}{2}+\frac{I_{f} C_{3} b R_{i}}{2}\right\}, \\
\xi_{6}(a, b, 0)=C_{1}+\left\{\frac{a R^{2}}{2}+\frac{b R^{3}}{6}\right\}\left(I_{f} C_{3}-I_{c} P\right) \\
\frac{d^{2} Z_{1 i}}{d T^{2}}=\left[\frac{2 I_{c} P}{T^{3}}\left\{\frac{a R_{i}^{2}}{2}+\frac{b R_{i}^{3}}{6}\right\}\right. \\
\quad-C_{2}\left\{\frac{1}{2 K}\left(\frac{3 b^{2} T}{2}+2 a b\right)\right. \\
\left.\quad+\frac{2 b}{3}-\frac{2 a b}{K}-\frac{3 b^{2} T}{2 K}\right\} \\
=\frac{1}{T^{3}}\left[2 \xi_{3}(a, b, 0) T^{4}+\xi_{4}(a, b, 0) T^{3}-2 \xi_{6}(a, b, 0)\right] .
\end{gathered}
$$

Now,

$$
\begin{aligned}
\frac{d Z_{2 i}}{d T}= & {\left[\left(P-C_{3}\right)\left(\frac{b}{2}\right)-I_{c} P\left\{\frac{b R_{i}}{2}-\frac{a}{2}-\frac{2 b T}{3}\right\}\right.} \\
& -C_{2}\left\{\frac{1}{2 K}\left(a^{2}+\frac{3 b^{2} T^{2}}{4}+2 a b T\right)+\frac{a}{2}+\frac{2 b T}{3}\right. \\
& \left.\left.-\frac{a^{2}}{K}-\frac{2 a b T}{K}-\frac{3 b^{2} T^{2}}{4 K}\right\}+\frac{C_{1}}{T^{2}}\right] \\
= & \frac{1}{T^{2}}\left(\xi_{3}(a, b, 0) T^{4}+\xi_{8}(a, b, 0) T^{3}+\xi_{5}(a, b) T^{2}+C_{1}\right),
\end{aligned}
$$

where

$$
\begin{gathered}
\xi_{8}(a, b, 0)=\left\{\frac{C_{2} a b}{K}-\left(C_{2}+I_{c} P\right) \frac{2 b}{3}\right\}, \\
\frac{d^{2} Z_{2 i}}{d T^{2}}=\left[I_{c} P\left\{\frac{2 b}{3}\right\}\right. \\
-C_{2}\left\{\frac{1}{2 K}\left(\frac{3 b^{2} T}{2}+2 a b\right)\right. \\
\left.\left.+\frac{2 b}{3}-\frac{2 a b}{K}-\frac{3 b^{2} T}{2 K}\right\}-\frac{2 C_{1}}{T^{3}}\right] \\
=\frac{1}{T^{3}}\left[2 \xi_{3}(a, b, 0) T^{4}+\xi_{8}(a, b, 0) T^{3}-2 C_{1}\right] .
\end{gathered}
$$

Proof of Lemma 3. The first part of the lemma is obvious from the formulas of $d Z_{1 i} / d T$ and $d^{2} Z_{1 i} / d T^{2}$. For the second part, if $Z_{1 i}$ does not have any stationary points in $\left[R_{i}, \infty\right)$, then $Z_{1 i}$ is either a monotonic increasing or monotonic decreasing function of $T \in\left[R_{i}, \infty\right)$. Here, $d Z_{1 i} / d T \rightarrow \infty$ as $T \rightarrow \infty$ because $\xi_{3}(a, b, 0)>0$. Hence, $Z_{1 i}$ is monotonic increasing if $\left[\left(R_{i}\right)^{4} \xi_{3}(a, b, 0)+\left(R_{i}\right)^{3} \xi_{4}(a, b, 0)+\left(R_{i}\right)^{2} \xi_{5}(a, b)+\xi_{6}(a, b, 0)\right]>0$ as $Z_{1 i}$ does not have stationary points in $\left[R_{i}, \infty\right)$. Here $t_{1}=$ $(1 / K)\left(a T+(b / 2) T^{2}\right)$ and $t_{1}$ increases with increasing value of $T$. According to our model, $t_{1}<R_{i}$; hence for the feasibility of the model, $t_{1}^{*}=R_{i}$ and $T^{*}$ is obtained from $(b / 2)\left(T^{*}\right)^{2}+$ $a\left(T^{*}\right)=K R_{i}$. By Descarte's rule $(b / 2)\left(T^{*}\right)^{2}+a\left(T^{*}\right)-K R_{i}=0$ may have atmost one positive root and one negative root. Now the positive root of the above equation is $T^{*}=(-a+$ $\left.\sqrt{a^{2}+2 K b R_{i}}\right) / b$. Here $T^{*}$ is unique by Descarte's rule. For feasibility of our model, $T^{*}$ must be greater than or equal to $R_{i}$. Therefore, $T^{*} \geq R_{i} \Rightarrow-a+\sqrt{a^{2}+2 K b R_{i}} \geq b R_{i} \Rightarrow$ $\sqrt{a^{2}+2 K b R_{i}} \geq a+b R_{i} \Rightarrow a^{2}+2 K b R_{i} \geq\left(a+b R_{i}\right)^{2} \Rightarrow a^{2}+$ $2 K b R_{i}-a^{2}-2 a b R_{i}-b^{2}\left(R_{i}\right)^{2} \geq 0 \Rightarrow 2(K-a) / b \geq R_{i}$. Thus $Z_{1 i}$ has a unique maximum value at $T=\left(-a+\sqrt{a^{2}+2 K b R_{i}}\right) / b$ if $R_{i} \leq 2(K-a) / b$ holds, hence the proof.

Proof of Lemma 4. The first part of the lemma is obvious (see the expressions of $d Z_{1 i} / d T$ and $d^{2} Z_{1 i} / d T^{2}$ ). For 
the second part, if $Z_{2 i}$ does not have any stationary points in $\left[0, R_{i}\right]$, then $Z_{2 i}$ is either monotonic increasing or monotonic decreasing function of $T \in\left[0, R_{i}\right]$. Here, $d Z_{2 i} / d T \rightarrow \infty$ as $T \rightarrow 0$ because $C_{1}>0$. So, $Z_{2 i}$ is monotonic increasing if $\left(1 /\left(R_{i}\right)^{2}\right)\left[\left(R_{i}\right)^{4} \xi_{3}(a, b, 0)+\left(R_{i}\right)^{3} \xi_{8}(a, b, 0)+\left(R_{i}\right)^{2} \xi_{5}(a, b)+\right.$ $\left.C_{1}\right]>0$ as $Z_{2 i}$ does not have stationary points in $\left[0, R_{i}\right]$. Hence, $Z_{2 i}$ has a maximum value when $T=R_{i}$ if $\left[\left(R_{i}\right)^{4} \xi_{3}(a, b, 0)+\left(R_{i}\right)^{3} \xi_{8}(a, b, 0)+\left(R_{i}\right)^{2} \xi_{5}(a, b)+C_{1}\right]>0$. Hence the proof.

\section{Derivation of Lemmas 5 and 6}

Differentiating (19), we have

$$
\begin{gathered}
\frac{d Z_{1 i}}{d T}=\left[-\frac{I_{c} P}{T^{2}}\left\{\frac{a R_{i}^{2}}{2}\right\}-C_{2}\left\{\frac{a}{2}-\frac{a^{2}}{2 K}\right\}\right. \\
\left.-I_{f} C_{3}\left\{\frac{a}{2}-\frac{a R_{i}^{2}}{2 T^{2}}\right\}+\frac{C_{1}}{T^{2}}\right], \\
\frac{d^{2} Z_{1 i}}{d T^{2}}=\left[\frac{I_{c} P}{T^{3}}\left\{a R_{i}^{2}\right\}-I_{f} C_{3}\left\{\frac{a R_{i}^{2}}{T^{3}}\right\}-\frac{2 C_{1}}{T^{3}}\right], \\
\frac{d Z_{2 i}}{d T}=\left[I_{c} P\left\{\frac{a}{2}\right\}-C_{2}\left\{\frac{a}{2}-\frac{a^{2}}{2 K}\right\}+\frac{C_{1}}{T^{2}}\right], \\
\frac{d^{2} Z_{2 i}}{d T^{2}}=\left[-\frac{2 C_{1}}{T^{3}}\right] .
\end{gathered}
$$

Proof of Lemma 5. We have $d Z_{1 i} / d T=\left[-\left(I_{c} P / T^{2}\right)\left\{\left(a R_{i}^{2}\right) /\right.\right.$ $\left.2\}-C_{2}\left\{a / 2-a^{2} / 2 K\right\}-\left(I_{f} C_{3}\right)\left\{a / 2-a R_{i}^{2} / 2 T^{2}\right\}+C_{1} / T^{2}\right]$ and $d^{2} Z_{1 i} / d T^{2}=\left[\left(I_{c} X / T^{3}\right)\left\{a R_{i}^{2}\right\}-I_{f} C_{3}\left\{a R^{2} / T^{3}\right\}-2 C_{1} / T^{3}\right]$. For maximization, $d Z_{1 i} / d T=0$. This implies that $T=$ $\left[K\left\{2 C_{1}+a R_{i}^{2}\left(I_{f} C_{3}-I_{c} P\right)\right\} /\left(C_{2} a(K-a)+I_{f} C_{3} a K\right)\right]^{1 / 2}=$ $T^{*}$ (say). At $T=T^{*}, d^{2} Z_{1 i} / d T^{2}=-(1 / K)\left[\left(C_{2} a(K-a)+\right.\right.$ $\left.\left.I_{f} C_{3} a K\right) / K\left\{2 C_{1}+a R_{i}^{2}\left(I_{f} C_{3}-I_{c} P\right)\right\}\right]^{1 / 2}$. If $K\left\{2 C_{1}+a R_{i}^{2}\left(I_{f} C_{3}-\right.\right.$ $\left.\left.I_{c} P\right)\right\}>0$, then $d^{2} Z_{1 i} / d T^{2}<0$. Again for the feasibility of the model, $t_{1}=a T / K<R_{i} \Rightarrow T<\left(K R_{i}\right) / a$ and $T>R_{i}$. Combining these, we have $R_{i}<T<K R_{i} / a$. That is, $R_{i}^{2}<$ $T^{2}<\left(K R_{i}\right)^{2} / a^{2}$. Hence at $T=T^{*}=\left[K\left\{2 C_{1}+a R_{i}^{2}\left(I_{f} C_{3}-\right.\right.\right.$ $\left.\left.\left.I_{c} P\right)\right\} /\left(C_{2} a(K-a)+I_{f} C_{3} a K\right)\right]^{1 / 2}, Z_{1 i}$ has a maximum value if $R_{i}^{2}<K\left\{2 C_{1}+a R_{i}^{2}\left(I_{f} C_{3}-I_{c} P\right)\right\} /\left(C_{2} a(K-a)+I_{f} C_{3} a K\right)<$ $K^{2} R_{i}^{2} / a^{2}$ hold, hence the proof.

Proof of Lemma 6. We have $d Z_{2 i} / d T=\left[I_{c} P(a / 2)-C_{2}\{a / 2-\right.$ $\left.\left.a^{2} / 2 K\right\}+C_{1} / T^{2}\right]$ and $d^{2} Z_{2 i} / d T^{2}=\left[-2 C_{1} / T^{3}\right]$. For optimization $d Z_{2 i} / d T=0$. This gives $T=\left[2 C_{1} K /\left(C_{2} a(K-\right.\right.$ a) $\left.\left.-I_{c} P a K\right)\right]^{1 / 2}$. For the real values of $T, C_{2} a(K-a)-I_{c} P a K>0$ and also for the feasibility of the model, $T<R_{i}$ which imply that $T^{2}<\left(R_{i}\right)^{2} \Rightarrow 0<2 C_{1} K /\left(C_{2} a(K-a)-I_{c} P a K\right)<R_{i}^{2}$. The second derivative of $Z_{2 i}$ at $T=T^{*}$ is always negative. Therefore $Z_{2 i}$ has a global maximum at $T=T^{*}=\left[2 C_{1} K /\right.$ $\left.\left(C_{2} a(K-a)-I_{c} P a K\right)\right]^{1 / 2}$ if $0<2 C_{1} K /\left(C_{2} a(K-a)-I_{c} P a K\right)<$ $R_{i}^{2}$ hold, hence the lemma.

\section{Derivation of Lemmas 7 and 8}

Now differentiating $Z_{1 i}$ and $Z_{2 i}$ with respect to $T$, we obtain

$$
\begin{aligned}
& \frac{d Z_{1 i}}{d T}=\frac{a}{b T}\left[\left(P-C_{3}\right)\left(b \exp (b T)-\frac{(\exp (b T)-1)}{T}\right)\right. \\
& +\frac{I_{c} P}{T}\left\{R_{i}+\frac{1}{b}\left(1-\exp \left(b R_{i}\right)\right)\right\} \\
& -C_{2}\left\{\frac{a \exp (b T)}{K}-\frac{a \exp (2 b T)}{K}\right. \\
& +T b \exp (b T)-\frac{a \exp (b T)}{K b T} \\
& +\frac{a \exp (2 b T)}{2 T K b}-\frac{1}{b T} \\
& \left.+\frac{a}{2 K b T}-\exp (b T)+\frac{\exp (b T)}{b T}\right\} \\
& -I_{f} C_{3}\left\{\left(T-R_{i}\right) b \exp (b T)\right. \\
& -\frac{\left(T-R_{i}\right) \exp (b T)}{T} \\
& \left.\left.+\frac{\exp (b T)-\exp \left(b R_{i}\right)}{b T}\right\}+\frac{b C_{1}}{a T}\right]
\end{aligned}
$$

Using $d Z_{1 i} / d T=0$,

$$
\begin{aligned}
\frac{d^{2} Z_{1 i}}{d T^{2}} & \\
=\frac{a}{b T}[ & \left(P-C_{3}\right) \\
& \times\left(b^{2} \exp (b T)-\frac{T b \exp (b T)-\exp (b T)+1}{T^{2}}\right) \\
& -\frac{I_{c} P}{T^{2}}\left\{R_{i}+\frac{1}{b}\left(1-\exp \left(b R_{i}\right)\right)\right\} \\
- & C_{2}\left\{\begin{array}{c}
\frac{a b \exp (b T)}{K}-\frac{2 a b \exp (2 b T)}{K} \\
+T b^{2} \exp (b T)-\frac{a \exp (b T)}{K T}+\frac{a \exp (b T)}{K b T^{2}} \\
+\frac{a \exp (2 b T)}{K T}-\frac{a \exp (2 b T)}{2 T^{2} K b}+\frac{1}{b T^{2}} \\
\left.-\frac{a}{2 K b T^{2}}+\frac{\exp (b T)}{T}-\frac{\exp (b T)}{b T^{2}}\right\}
\end{array}\right.
\end{aligned}
$$




$$
\begin{aligned}
-I_{f} C_{3}\{ & \left(T-R_{i}\right) b^{2} \exp (b T) \\
& -\frac{R_{i}(T b \exp (b T)-\exp (b T))}{T^{2}} \\
& \left.+\frac{T b \exp (b T)-\exp (b T)+\exp \left(b R_{i}\right)}{b T^{2}}\right\} \\
\left.-\frac{b C_{1}}{a T^{2}}\right] &
\end{aligned}
$$

From Case 2, we have

$$
\begin{aligned}
& \frac{d Z_{2 i}}{d T}=\frac{a}{b T}\left[\left(P-C_{3}\right)\right.\left(b \exp (b T)-\frac{(\exp (b T)-1)}{T}\right) \\
&-\frac{I_{c} P}{T}\{ T b \exp (b T)-R_{i} b \exp (b T) \\
&-\frac{1}{b T}(1-\exp (b T)) \\
&\left.+\frac{R_{i} \exp (b T)}{T}-\frac{R_{i}}{T}-\exp (b T)\right\} \\
&-C_{2}\left\{\begin{array}{l}
\frac{a \exp (b T)}{K}-\frac{a \exp (2 b T)}{K} \\
+
\end{array}\right. \\
& \quad+\frac{a \exp (2 b T)}{2 T K b}-\frac{1}{b T}+\frac{a}{2 K b T} \\
&\left.\left.\quad-\exp (b T)+\frac{\exp (b T)}{b T}\right\}+\frac{b C_{1}}{a T}\right] .
\end{aligned}
$$

Using $d Z_{2 i} / d T=0$,

$$
\begin{aligned}
& \frac{d^{2} Z_{2 i}}{d T^{2}} \\
& =\frac{a}{b T}\left[\left(P-C_{3}\right)\right. \\
& \quad \times\left(b^{2} \exp (b T)-\frac{T b \exp (b T)-\exp (b T)+1}{T^{2}}\right) \\
& \quad-I_{c} P\left\{T b^{2} \exp (b T)-R_{i} b^{2} \exp (b T)-\frac{\exp (b T)}{T}\right. \\
& \quad+\frac{1}{b T^{2}}-\frac{\exp (b T)}{b T^{2}}+\frac{R_{i} b \exp (b T)}{T} \\
& \left.\quad-\frac{R_{i} \exp (b T)}{T^{2}}+\frac{R_{i}}{T^{2}}\right\}
\end{aligned}
$$

$$
\begin{gathered}
-C_{2}\left\{\frac{a b \exp (b T)}{K}-\frac{2 a b \exp (2 b T)}{K}+T b^{2} \exp (b T)\right. \\
-\frac{a \exp (b T)}{K T}+\frac{a \exp (b T)}{K b T^{2}}+\frac{a \exp (2 b T)}{K T} \\
-\frac{a \exp (2 b T)}{2 T^{2} K b}+\frac{1}{b T^{2}}-\frac{a}{2 K b T^{2}} \\
\left.\left.+\frac{\exp (b T)}{T}-\frac{\exp (b T)}{b T^{2}}\right\}-\frac{b C_{1}}{a T^{2}}\right] .
\end{gathered}
$$

Proof of Lemma 7. The first part of the lemma is obvious. For the second part, if $Z_{1 i}$ does not have any stationary points in $\left[R_{i}, \infty\right)$, then $Z_{1 i}$ is either monotonic increasing or monotonic decreasing function of $T \in\left[R_{i}, \infty\right)$. Here, $d Z_{1 i} / d T \rightarrow$ $\infty$ as $T \rightarrow \infty$ because

$$
\begin{aligned}
& \frac{d Z_{1 i}}{d T} \\
& =\frac{a}{b T}\left[\left(P-C_{3}\right)\left(b \exp (b T)-\frac{(\exp (b T)-1)}{T}\right)\right. \\
& +\frac{I_{c} P}{T}\left\{R_{i}+\frac{1}{b}\left(1-\exp \left(b R_{i}\right)\right)\right\} \\
& -C_{2}\left\{\frac{a \exp (b T)}{K}-\frac{a \exp (2 b T)}{K}+T b \exp (b T)\right. \\
& -\frac{a \exp (b T)}{K b T}+\frac{a \exp (2 b T)}{2 T K b}-\frac{1}{b T} \\
& \left.+\frac{a}{2 K b T}-\exp (b T)+\frac{\exp (b T)}{b T}\right\} \\
& -I_{f} C_{3}\left\{\left(T-R_{i}\right) b \exp (b T)-\frac{\left(T-R_{i}\right) \exp (b T)}{T}\right. \\
& \left.\left.+\frac{\exp (b T)-\exp \left(b R_{i}\right)}{b T}\right\}+\frac{b C_{1}}{a T}\right] \\
& =\frac{a \exp (b T)}{b T}\left[\left(P-C_{3}\right)\left(b-\frac{1}{T}\right)\right. \\
& -C_{2}\left\{\frac{a}{K}+T b-\frac{a}{K b T}-1+\frac{1}{b T}\right\} \\
& \left.-I_{f} C_{3}\left\{\left(T-R_{i}\right) b+\frac{R_{i}}{T}+\frac{1}{b T}\right\}\right] \\
& +\frac{a}{b T}\left[\frac{\left(P-C_{3}\right)}{T}+\frac{I_{c} P}{T}\left\{R_{i}+\frac{1}{b}\right\}\right. \\
& \left.-C_{2}\left\{\frac{a}{2 K b T}-\frac{1}{b T}\right\}+I_{f} C_{3} \frac{\exp \left(b R_{i}\right)}{b T}\right] \\
& +\frac{a}{b T}\left[-C_{2}\left\{-\frac{a}{K}+\frac{a}{2 T K b}\right\}\right] \exp (2 b T)+\frac{C_{1}}{T^{2}} \text {. }
\end{aligned}
$$


Using L'Hospital's rule

$$
\begin{aligned}
\lim _{T \rightarrow \infty} \frac{d Z_{1 i}}{d T} & =\lim _{T \rightarrow \infty} \frac{a \exp (b T)}{b T^{2}} \\
& \times\left[\left(P-C_{3}\right)(b T-1)\right. \\
& \quad-C_{2}\left\{\frac{a T}{K}+T^{2} b-\frac{a}{K b}-T+\frac{1}{b}\right\} \\
& +\lim _{T \rightarrow \infty} \frac{a}{b T^{2}}\left[\left(P-C_{3}\right)+I_{c} P\left\{R_{i}+\frac{1}{b}\right\}\right. \\
\simeq & \frac{a}{b} \lim _{T \rightarrow \infty} \frac{G(T)}{T^{2}} \operatorname{since}|G(T)|>|F(T)| \\
= & \left.\left.\left.\frac{a}{b} \lim _{T \rightarrow \infty} \frac{G^{\prime}(T)}{2 T}=\frac{a}{2 b} \lim _{T \rightarrow \infty} G^{\prime \prime}(T)-R_{i}\right)+\frac{1}{b}\right\}\right] \\
& +\lim _{T \rightarrow \infty} \frac{a}{b T^{2}} \exp (2 b T)\left[-C_{2}\left\{-\frac{a T}{K}+\frac{a}{2 K b}\right\}\right] \\
& +\lim _{T \rightarrow \infty} \frac{C_{1}}{T^{2}}=\frac{a}{b} \lim _{T \rightarrow \infty} \frac{F(T)}{T^{2}}+\frac{a}{b} \lim _{T \rightarrow \infty} \frac{G(T)}{T^{2}}
\end{aligned}
$$

where

$$
\begin{aligned}
& F(T)=[\exp (b T)[\left(P-C_{3}\right)(b T-1) \\
&-C_{2}\left\{\frac{a T}{K}+T^{2} b-\frac{a}{K b}-T+\frac{1}{b}\right\} \\
&\left.-I_{f} C_{3}\left\{\left(T-R_{i}\right) T b+R_{i}+\frac{1}{b}\right\}\right] \\
&+ {\left[\frac{\left(P-C_{3}\right)}{T}+I_{c} P\left\{R_{i}+\frac{1}{b}\right\}\right.} \\
&\left.-C_{2}\left\{\frac{a}{2 K b}-\frac{1}{b}\right\}+I_{f} C_{3} \frac{\exp \left(b R_{i}\right)}{b}\right] \\
&\left.+\frac{C_{1}}{T^{2}}\right], \quad \\
& G(T)=-\frac{a C_{2}}{2 K b} \exp (2 b T)(1-2 b T) .
\end{aligned}
$$

Now,

$$
\begin{aligned}
G(T) & =-\frac{a C_{2}}{2 K b} \exp (2 b T)(1-2 b T) \Longrightarrow G^{\prime}(T) \\
& =\frac{2 a b T C_{2}}{K} \exp (2 b T) \Longrightarrow G^{\prime \prime}(T) \\
& =\frac{(2 b+1) 2 a b C_{2}}{K} \exp (2 b T) \Longrightarrow G^{\prime \prime}(T) \longrightarrow+\infty .
\end{aligned}
$$

Hence, $Z_{1 i}$ is monotonic increasing if $\left.\left(d Z_{1 i} / d T\right)\right|_{T=R_{i}}>0$ as $Z_{1 i}$ does not have stationary points in $\left[R_{i}, \infty\right)$. Here, $t_{1}=$ $(a / K b)(\exp (b T)-1)$ and $t_{1}$ increases with increasing value of $T$. As in our model, $t_{1}<R_{i}$; hence, for the feasibility of the model, $t_{1}^{*}=R_{i}$ and $T^{*}$ is obtained from $(a / b)\left(\exp \left(b T^{*}\right)-1\right)=$ $K R_{i}$. Therefore, $Z_{1 i}$ has a maximum value at $T^{*}=(1 / b) \ln (1+$ $\left.K b R_{i} / a\right)$ if $\left.\left(d Z_{1 i} / d T\right)\right|_{T=R_{i}}>0$. Here $T^{*}$ is unique, hence the proof.

Proof of Lemma 8. The first part of the lemma is obvious. For the second part, if $Z_{2 i}$ does not have any stationary points in $\left[0, R_{i}\right]$, then $Z_{2 i}$ is either monotonic increasing or monotonic decreasing function of $T \in\left[0, R_{i}\right]$. Here $d Z_{2 i} / d T \rightarrow \infty$ as $T \rightarrow 0$ because

$$
\begin{aligned}
& \frac{d Z_{2 i}}{d T} \\
& =\frac{a}{b T}\left[\left(P-C_{3}\right)\left(b \exp (b T)-\frac{(\exp (b T)-1)}{T}\right)\right. \\
& -\frac{I_{c} P}{T}\left\{T b \exp (b T)-R_{i} b \exp (b T)\right. \\
& -\frac{1}{b T}(1-\exp (b T))+\frac{R_{i} \exp (b T)}{T} \\
& \left.-\frac{R_{i}}{T}-\exp (b T)\right\} \\
& -C_{2}\left\{\frac{a \exp (b T)}{K}-\frac{a \exp (2 b T)}{K}\right. \\
& +T b \exp (b T)-\frac{a \exp (b T)}{K b T} \\
& +\frac{a \exp (2 b T)}{2 T K b}-\frac{1}{b T}+\frac{a}{2 K b T} \\
& \left.\left.-\exp (b T)+\frac{\exp (b T)}{b T}\right\}+\frac{b C_{1}}{a T}\right] \text {. }
\end{aligned}
$$

Using L'Hospital's Rule, we obtain

$$
\begin{aligned}
& \lim _{T \rightarrow 0} \frac{d Z_{2 i}}{d T} \\
&=\lim _{T \rightarrow 0} \frac{a}{b T^{3}}
\end{aligned}
$$




$$
\begin{aligned}
\times\left[\left(P-C_{3}\right)\right. & \{b T \exp (b T)-(\exp (b T)-1)\} T \\
-I_{c} P\{ & T\left(T b \exp (b T)-R_{i} b \exp (b T)\right) \\
& -\frac{1}{b}(1-\exp (b T))+R_{i} \exp (b T) \\
& \left.-R_{i}-T \exp (b T)\right\} \\
-C_{2}\{ & T\left(\frac{a \exp (b T)-a \exp (2 b T)}{K}+T b \exp (b T)\right) \\
& -\frac{2 a \exp (b T)-a \exp (2 b T)}{2 K b}-\frac{1}{b}+\frac{a}{2 K b} \\
& \left.\left.-T \exp (b T)+\frac{\exp (b T)}{b}\right\}+\frac{b C_{1} T}{a}\right] \\
=\lim _{T \rightarrow 0} \frac{H(T)}{T^{3}}= & \lim _{T \rightarrow 0} \frac{H^{\prime}(T)}{3 T^{2}} \longrightarrow+\infty,
\end{aligned}
$$

where

$$
\begin{aligned}
& H(T) \\
& \begin{aligned}
\frac{a}{b}\left[\left(P-C_{3}\right)\right. \\
\quad \times\{b T \exp (b T)-(\exp (b T)-1)\} T \\
-I_{c} P\left\{T\left(T b \exp (b T)-R_{i} b \exp (b T)\right)\right. \\
\quad-\frac{1}{b}(1-\exp (b T))+R_{i} \exp (b T) \\
\left.-R_{i}-T \exp (b T)\right\} \\
-C_{2}\left\{T\left(\frac{a \exp (b T)-a \exp (2 b T)}{K}+T b \exp (b T)\right)\right. \\
-\frac{2 a \exp (b T)-a \exp (2 b T)}{2 K b}-\frac{1}{b}+\frac{a}{2 K b} \\
\left.\left.-T \exp (b T)+\frac{\exp (b T)}{b}\right\}+\frac{b C_{1} T}{a}\right] .
\end{aligned}
\end{aligned}
$$

Now,

$$
\begin{aligned}
& H^{\prime}(T) \\
& =\frac{a}{b}\left[\left(P-C_{3}\right)\right. \\
& \quad \times\left\{b T \exp (b T)-\exp (b T)+1+T^{2} b^{2} \exp (b T)\right\} \\
& \quad-I_{c} P\left\{T b \exp (b T)+T^{2} b^{2} \exp (b T)\right. \\
& \left.-R_{i} T b^{2} \exp (b T)\right\}
\end{aligned}
$$

$$
\begin{gathered}
-C_{2}\left\{T\left(\frac{a \exp (b T)-a \exp (2 b T)}{K}-\exp (b T)\right)\right. \\
-T^{2}\left(\frac{a b \exp (b T)-2 a b \exp (2 b T)}{K}\right. \\
\quad+2 b \exp (b T))+T^{3} b^{2} \exp (b T) \\
-\frac{2 a \exp (b T)-a \exp (2 b T)}{2 K b} \\
\left.\left.-\frac{1}{b}+\frac{a}{2 K b}+\frac{\exp (b T)}{b}\right\}+\frac{b C_{1}}{a}\right] .
\end{gathered}
$$

Here, $\lim _{T \rightarrow 0} H^{\prime}(T)=C_{1}$ so that $Z_{1 i}$ is monotonic increasing if $\left.\left(d Z_{1 i} / d T\right)\right|_{T=R_{i}}>0$ as $Z_{1 i}$ does not have stationary points in $\left[0, R_{i}\right]$. Here, $t_{1}=(a / K b)(\exp (b T)-1)$, and $t_{1}$ increases with increasing value of $T$. According to our model, $t_{1}<R_{i}$; hence, for the feasibility of the model, $t_{1}^{*}=R_{i}$ and $T^{*}$ is obtained from $(a / b)\left(\exp \left(b T^{*}\right)-1\right)=K R_{i}$. Therefore, $Z_{1 i}$ has a maximum value at $T^{*}=(1 / b) \ln \left(1+K b R_{i} / a\right)$ if $\left.\left(d Z_{1 i} / d T\right)\right|_{T=R_{i}}>$ 0 . Here, $T^{*}$ is unique, hence the proof.

\section{Acknowledgments}

The authors want to acknowledge the reviewers for their constructive comments to revise the paper. The first author would like to express his heartiest gratitude to his parents, wife, and son for their valuable support during the research. This research is supported by the University Grant Commission, Delhi, India, from the research grant of the Minor Project (no. 41-1433/2012(SR)). The corresponding author wants to acknowledge the infrastructural assistance available at Vidyasagar University, West Bengal, India.

\section{References}

[1] F. W. Harris, "How many parts to make at once factory," The Magazine of Management, vol. 10, pp. 135-136, 1913.

[2] W. A. Donaldson, "Inventory replenishment policy for a linear trend in demand: an analytical solution," Operational Research Quarterly, vol. 28, pp. 663-670, 1977.

[3] S. K. Goyal, "On improving replenishment policies for linear trend in demand," Engineering Costs and Production Economics, vol. 10, no. 1, pp. 73-76, 1986.

[4] A. Goswami and K. S. Chaudhuri, "EOQ model for deteriorating items with shortages and a linear trend in demand," Journal of the Operational Research Society, vol. 42, no. 12, pp. 1105-1110, 1991.

[5] S. K. Goyal, D. Morin, and F. Nebebe, "Finite horizon trended inventory replenishment problem with shortages," Journal of the Operational Research Society, vol. 43, no. 12, pp. 1173-1178, 1992.

[6] M. A. Hariga and L. Benkherouf, "Optimal and heuristic inventory replenishment models for deteriorating items with exponential time-varying demand," European Journal of Operational Research, vol. 79, no. 1, pp. 123-137, 1994.

[7] H. M. Wee, "A deterministic lot-size inventory model for deteriorating items with shortages and a declining market," 
Computers and Operations Research, vol. 22, no. 3, pp. 345-356, 1995.

[8] S. Khanra and K. S. Chaudhuri, "A note on an order-level inventory model for a deteriorating item with time-dependent quadratic demand," Computers and Operations Research, vol. 30, no. 12, pp. 1901-1916, 2003.

[9] S. Sana and K. S. Chaudhuri, "On a volume flexible production policy for a deteriorating item with time-dependent demand and shortages," Advanced Modeling and Optimization, vol. 6, no. 1, pp. 57-74, 2004.

[10] L. E. Cárdenas-Barrón, "Optimal ordering policies in response to a discount offer: corrections," International Journal of Production Economics, vol. 122, pp. 783-789, 2009.

[11] B. Sarkar, S. S. Sana, and K. Chaudhuri, "An imperfect production process for time varying demand with inflation and time value of money-an EMQ model," Expert Systems with Applications, vol. 38, no. 11, pp. 13543-13548, 2011.

[12] P. L. Abad, "Determining optimal selling price and lot size when suppliers offers all unit quantity discounts," Decision Science, vol. 19, pp. 622-634, 1988.

[13] K. H. Kim and H. Hwang, "An incremental discount pricing schedule with multiple customers and single price break," European Journal of Operational Research, vol. 35, no. 1, pp. 7179, 1988.

[14] S. K. Goyal, "Economic order quantity under conditions of permissible delay in payments," Journal of the Operational Research Society, vol. 36, no. 4, pp. 335-338, 1985.

[15] S. P. Aggarwal and C. K. Jaggi, "Ordering policies of deterioration items under permissible delay in payments," Journal of the Operational Research Society, vol. 46, pp. 658-662, 1995.

[16] P. Chu, K.-J. Chung, and S.-P. Lan, "Economic order quantity of deteriorating items under permissible delay in payments," Computers and Operations Research, vol. 25, no. 10, pp. 817-824, 1998.

[17] A. M. M. Jamal, B. R. Sarker, and S. Wang, "Optimal payment time for a retailer under permitted delay of payment by the wholesaler," International Journal of Production Economics, vol. 66 , no. 1, pp. 59-66, 2000.

[18] J.-T. Teng, "On the economic order quantity under conditions of permissible delay in payments," Journal of the Operational Research Society, vol. 53, no. 8, pp. 915-918, 2002.

[19] F. J. Arcelus, N. H. Shah, and G. Srinivasan, "Retailer's pricing, credit and inventory policies for deteriorating items in response to temporary price/credit incentives," International Journal of Production Economics, vol. 81-82, pp. 153-162, 2003.

[20] Y.-F. Huang, "Economic order quantity under conditionally permissible delay in payments," European Journal of Operational Research, vol. 176, no. 2, pp. 911-924, 2007.

[21] Y.-F. Huang, "Optimal retailer's replenishment decisions in the EPQ model under two levels of trade credit policy," European Journal of Operational Research, vol. 176, no. 3, pp. 1577-1591, 2007.

[22] L. E. Cárdenas-Barrón, “The derivation of EOQ/EPQ inventory models with two backorders costs using analytic geometry and algebra," Applied Mathematical Modelling, vol. 35, no. 5, pp. 2394-2407, 2011.

[23] H.-M. Teng, P.-H. Hsu, Y. Chiu, and H. M. Wee, "Optimal ordering decisions with returns and excess inventory," Applied Mathematics and Computation, vol. 217, no. 22, pp. 9009-9018, 2011.
[24] B. Sarkar, "An EOQ model with delay in payments and stock dependent demand in the presence of imperfect production," Applied Mathematics and Computation, vol. 218, no. 17, pp. 8295-8308, 2012.

[25] B. Sarkar, "An EOQ model with delay in payments and time varying deterioration rate," Mathematical and Computer Modelling, vol. 55, no. 3-4, pp. 367-377, 2012.

[26] K. Forghani, A. Mirzazadeh, and M. Rafiee, "A price-dependent demand model in the single period inventory system with price adjustment," Journal of Industrial Engineering, vol. 2013, Article ID 593108, 9 pages, 2013. 

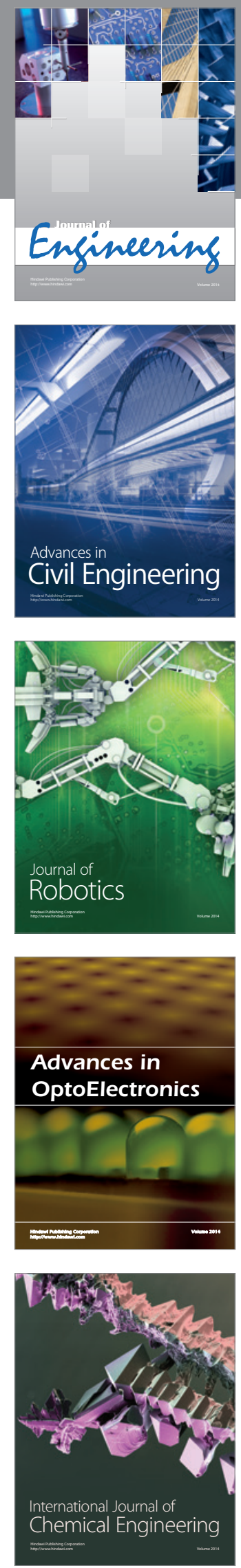

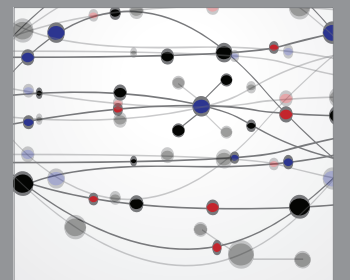

The Scientific World Journal
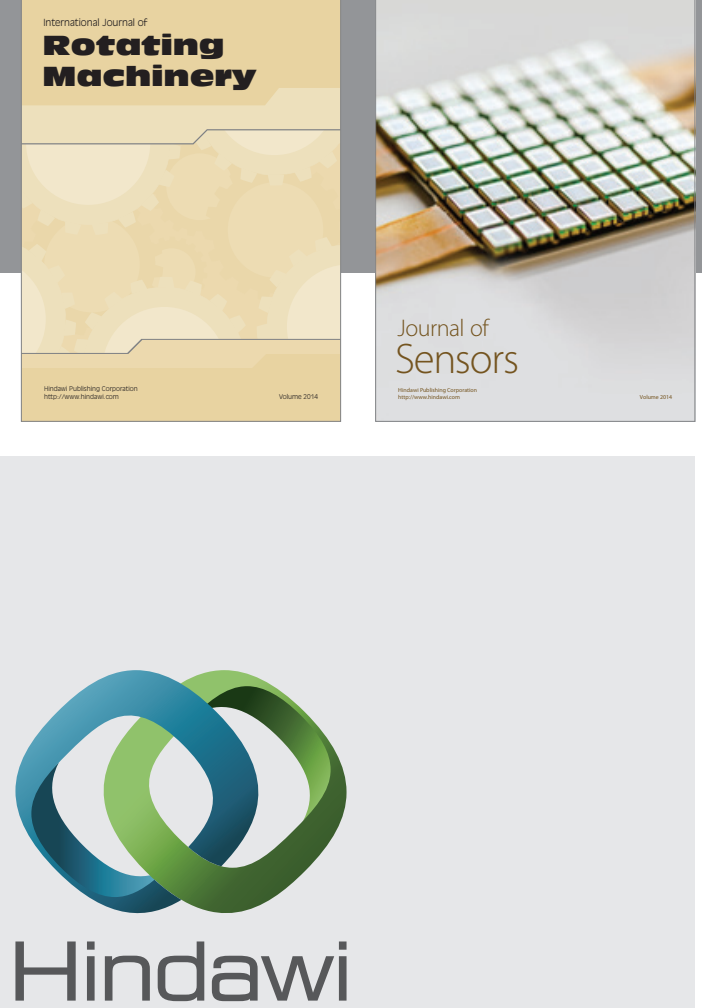

Submit your manuscripts at http://www.hindawi.com
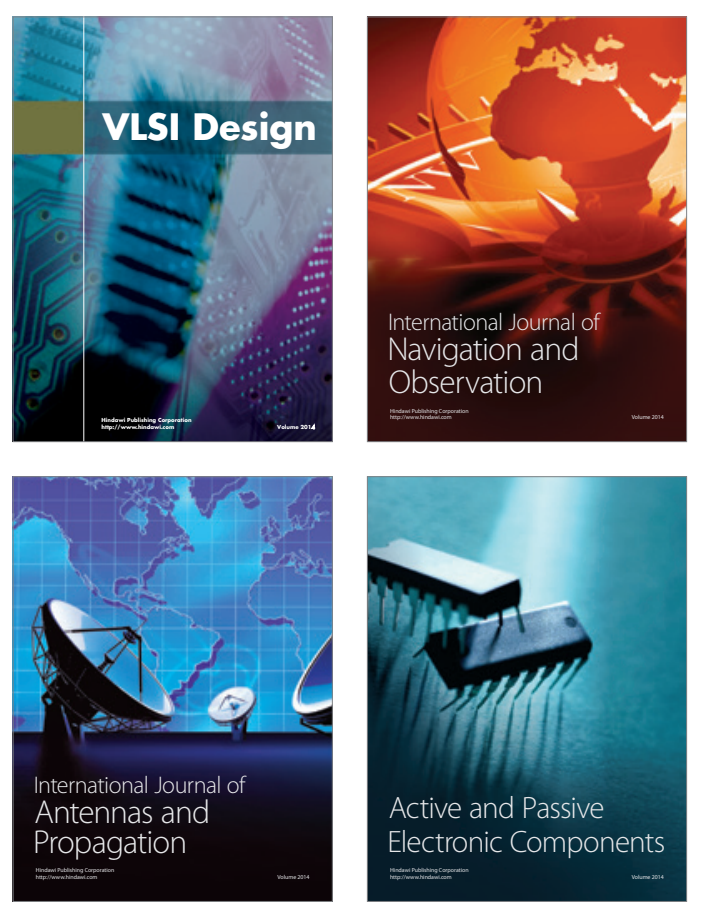
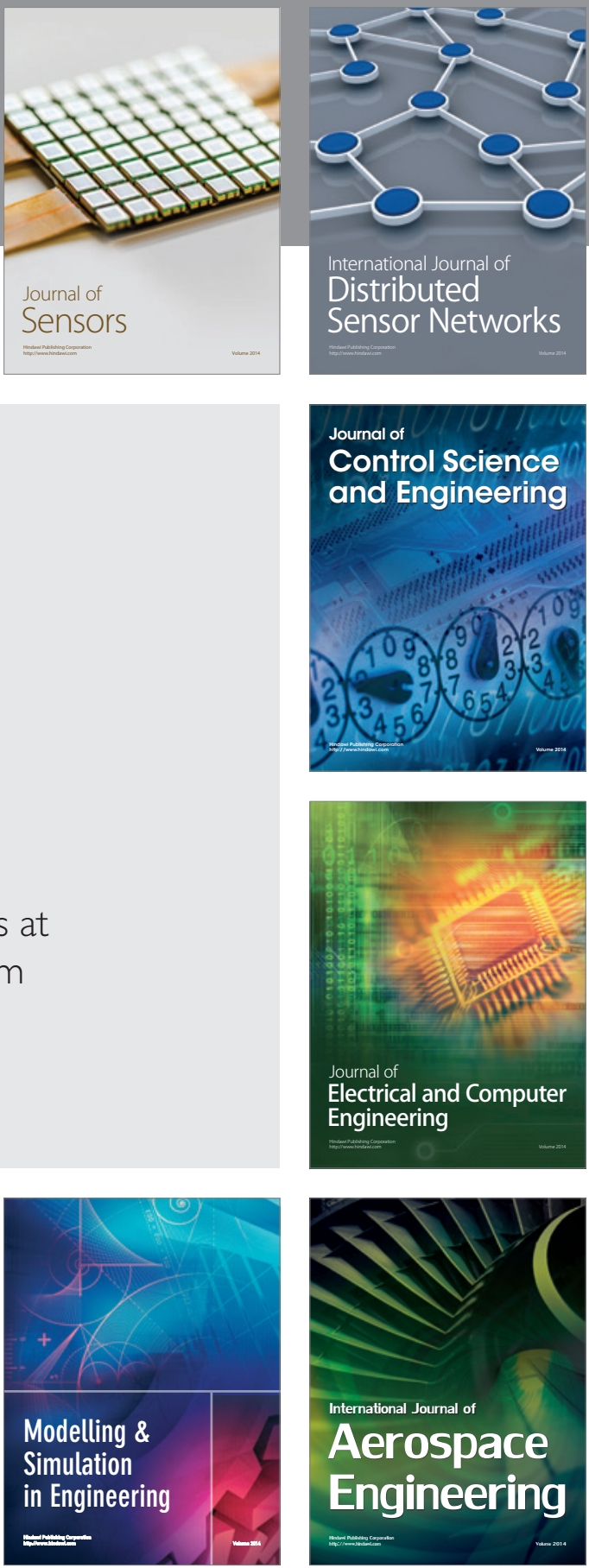

Journal of

Control Science

and Engineering
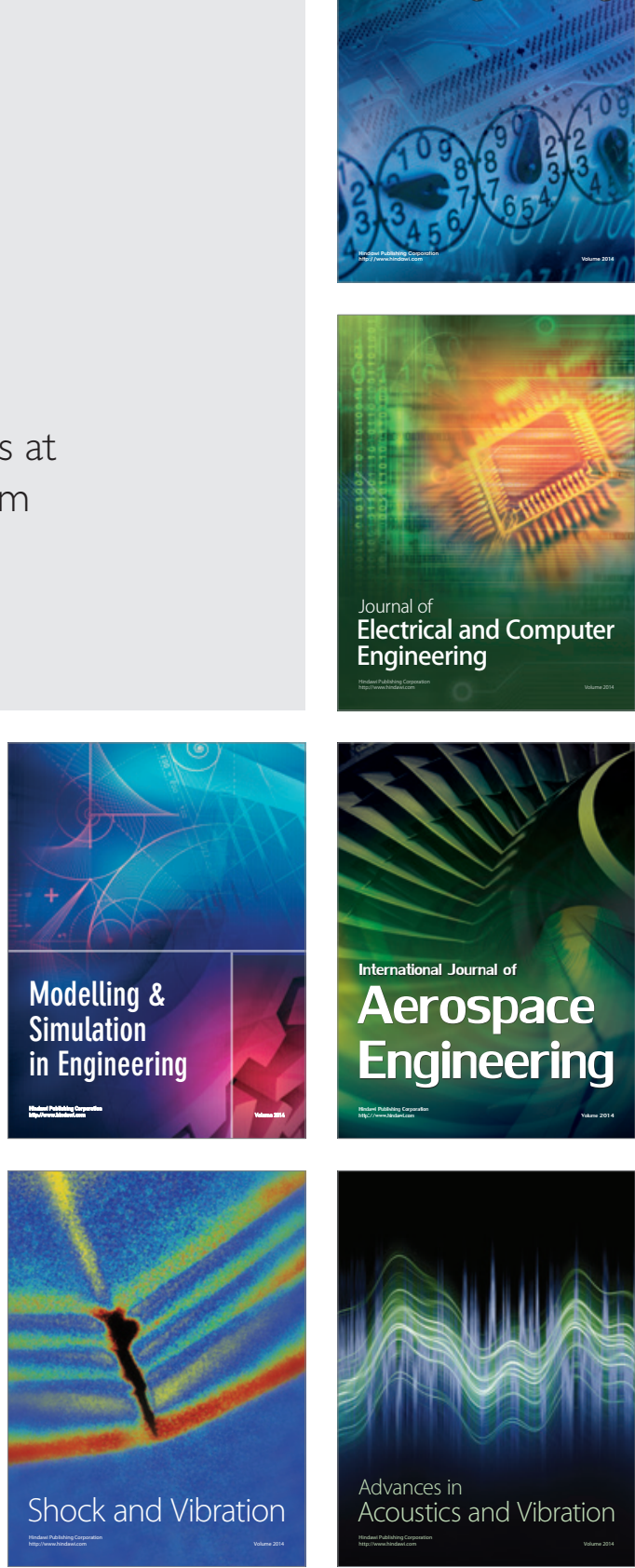